\title{
Incorporating Thruster Dynamics in the Control of an Underwater Vehicle
}

by

JOHN GORDON COOKE V

B.S. Mechanical Engineering, United States Naval Academy (1978)

Submitted in partial fulfillment of the

requirements for the degrees of

OCEAN ENGINEER

at the

MASSACHUSETTS INSTITUTE OF TECHNOLOGY

and the

WOODS HOLE OCEANOGRAPHIC INSTITUTION

and

MASTER OF SCIENCE IN OCEAN ENGINEERING

at the

MASSACHUSETTS INSTITUTE OF TECHNOLOGY

September 1989

(c) John G. Cooke V, 1989

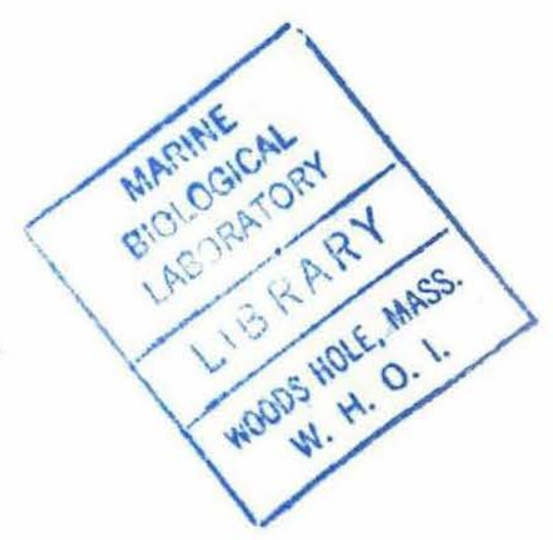

The author hereby grants to MIT and WHOI permission to reproduce and to distribute copies of this thesis documenting whole or in part. ?

Signature of Author

Joine Program in oceanographic Engineering

Massachusetts Institute of Technology

Woods Hole Oceanographic Institution

$1 \cap \cap$, August 11, 1989

Certified by

$\smile$

Dł. Dana k. Yoerger

Wogds Hole Oceanographic Institution

Accepted by

W.'Kendall Melville, Chairman

Joint Committee for Oceanographic Engineering

Massachusetts Institute of Technology

Woods Hole Oceanographic Institution 
$-2-$ 


\title{
Incorporating Thruster Dynamics in the Control of an Underwater Vehicle
}

\author{
by \\ JOHN GORDON COOKE V \\ Submitted to the Massachusetts Institute of Technology/ \\ Woods Hole Oceanographic Institution \\ Joint Program in Oceanographic Engineering \\ on August 11, 1989 in partial fulfillment of the \\ requirements for the degree of \\ OCEAN ENGINEER \\ and \\ MASTER OF SCIENCE IN OCEAN ENGINEERING
}

\begin{abstract}
The dynamics of an underwater vehicle are greatly influenced by the dynamics of the thrusters. Precise control, for example to perform repeatable survey or coordinated vehicle/manipulator control, should incorporate knowledge of thruster dynamic behavior. An energy-based lumped parameter model of the nonlinear thruster dynamic response is developed and experimentally verified using static and dynamic thruster relationships. Three controllers to compensate for the nonlinear dynamics are designed including analog lead compensation, model-based computed torque and adaptive sliding control techniques. The proposed controller designs are implemented and evaluated in a hybrid, one degree-of-freedom vehicle simulation using an actual thruster under digital control as the actuator. Controller evaluation and comparison is based on observed vehicle tracking performance.

The incorporation of thruster dynamics is shown to significantly improve vehicle tracking performance. Superior, robust tracking performance with significant model uncertainty is demonstrated in the application of the adaptive sliding control technique. The evaluated adaptive controller structure may permit on-line adaptation to complex hydrodynamic phenomena associated with complete vehicle/thruster configurations such as cross-flow and mutual interference.
\end{abstract}

Thesis Supervisor: $\quad$ Dr. Dana R. Yoerger

Associate Scientist

Woods Hole Oceanographic Institution 


\section{Acknowledgements}

The successful completion of this thesis and culmination of my graduate studies were made possible by the support and encouragement of my friends and colleagues. Special appreciation is due to,

Dr. Robert Ballard for his encouragement to perform my graduate work at the Deep Submergence Laboratory.

Dana Yoerger my advisor, for his unfailing confidence and friendship.

J-J. Slotine for his suggestions and guidance.

Hagen Schempf for his technical advice, friendship and most of all, his patience.

Franz Hover for his constant encouragement and willingness to listen.

My Children Jace, Jameson, Patty, and Julie for their patience, and love.

Deborah my wife, the "...wind beneath my wings!", to whom the thesis is dedicated.

The United States Navy is gratefully acknowledged for the graduate education opportunity provided. This research was also supported by OCNR contract N00014-86C-0038, N00014-88-K-2022, and ONT grant N00014-87-J-1111. 


\section{Author's Biographical Note}

LCDR John G. Cooke, USN completed his undergraduate studies at the U. S. Naval Academy, receiving a Bachelor of Science in Mechanical Engineering and was commissioned in the U. S. Navy in 1978. He completed nuclear propulsion and submarine training in 1980 and was assigned to the commissioning crew of the USS OHIO (SSBN 726). He served as Engineer Officer of the commissioning crew of the USS GEORGIA (SSBN 729) and as Navigator and Operations Officer of the USS SAN FRANCISCO (SSN 711) prior to his present assignment in the Massachusetts Institute of Technology/Woods Hole Oceanographic Institution Joint Program in Oceanographic Engineering.

LCDR Cooke's next assignment, following completion of his graduate studies is Officer-in-Charge of the U. S. Navy's nuclear-powered, deep submergence research vessel, NR-1. 


\section{Contents}

$\begin{array}{ll}\text { Chapter } 1 \text { INTRODUCTION } & 11\end{array}$

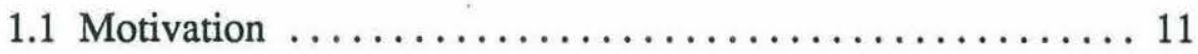

1.2 Research Objectives . . . . . . . . . . . . . 13

1.3 Outline of Thesis $\ldots \ldots \ldots \ldots \ldots \ldots \ldots \ldots \ldots \ldots \ldots \ldots$

$\begin{array}{ll}\text { Chapter } 2 \text { THRUSTER DYNAMICS } & 16\end{array}$

2.1 Lumped Parameter Model Development ............. 16

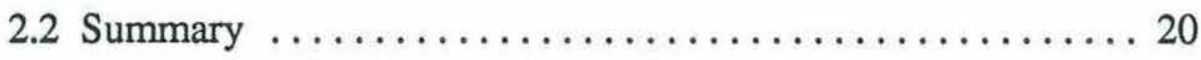

Chapter 3 THRUSTER MODEL VERIFICATION 23

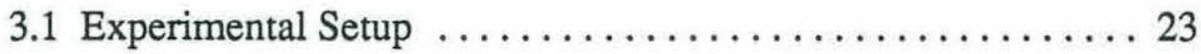

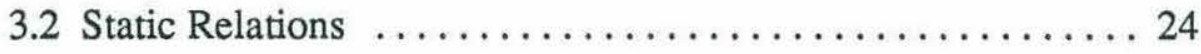

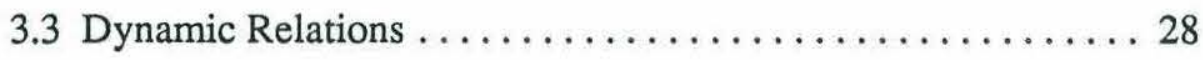

3.4 Parameter Comparisons $\ldots \ldots \ldots \ldots \ldots \ldots \ldots \ldots \ldots \ldots \ldots$

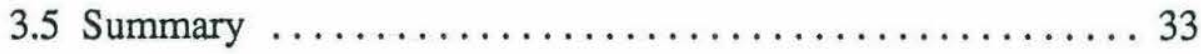

Chapter 4 CONTROLLER DESIGN 34

4.1 Lead Compensation ........................ 34

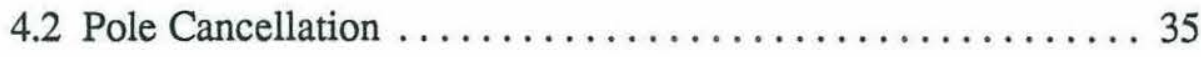

4.3 Sliding Controller $\ldots \ldots \ldots \ldots \ldots \ldots \ldots \ldots \ldots \ldots \ldots \ldots \ldots \ldots$

4.3.1 Adaptive Sliding Controller . . . . . . . . . . . 44 
4.4 Summary $\ldots \ldots \ldots \ldots \ldots \ldots \ldots \ldots \ldots \ldots \ldots \ldots \ldots, 46$

Chapter 5 CONTROLLER EVALUATION 49

5.1 Hybrid Simulation $\ldots \ldots \ldots \ldots \ldots \ldots \ldots \ldots \ldots \ldots \ldots \ldots \ldots \ldots$

5.1 .1 Vehicle Model $\ldots \ldots \ldots \ldots \ldots \ldots \ldots \ldots \ldots \ldots$

5.1 .2 Vehicle Position Controller ............... 51

5.1.3 Thruster Controller Interfaces $\ldots \ldots \ldots \ldots \ldots \ldots \ldots 51$

5.2 Evaluation Results $\ldots \ldots \ldots \ldots \ldots \ldots \ldots \ldots \ldots \ldots \ldots$

5.2 .1 No Compensation $\ldots \ldots \ldots \ldots \ldots \ldots \ldots \ldots \ldots$

5.2.2 Lead Compensation $\ldots \ldots \ldots \ldots \ldots \ldots \ldots \ldots \ldots$

5.2 .3 "Pole" Cancellation $\ldots \ldots \ldots \ldots \ldots \ldots \ldots \ldots \ldots \ldots .56$

5.2.4 Adaptive Sliding Controller .............. 61

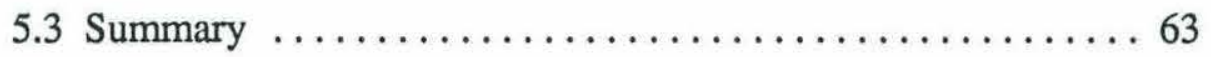

Chapter 6 EXPERIMENTAL SETUP 66

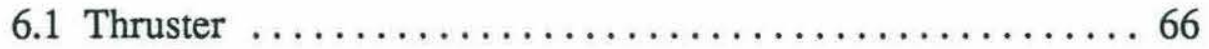

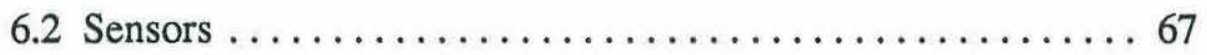

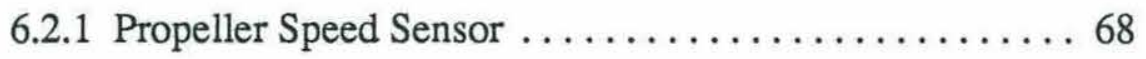

6.2.2 Thrust Sensor $\ldots \ldots \ldots \ldots \ldots \ldots \ldots \ldots \ldots \ldots \ldots$

6.2.3 Fluid Velocity Sensor $\ldots \ldots \ldots \ldots \ldots \ldots \ldots \ldots \ldots$

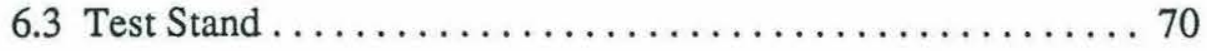

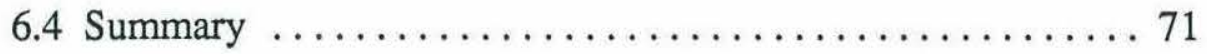

Chapter 7 SUMMARY, CONCLUSIONS AND

$\begin{array}{ll}\text { RECOMMENDATIONS } & 73\end{array}$ 


\section{Figures}

Figure 1.1: The remotely operated vehicle JASON. . . . . . . . . 12

Figure 2.1: Typical thruster schematic. ...............

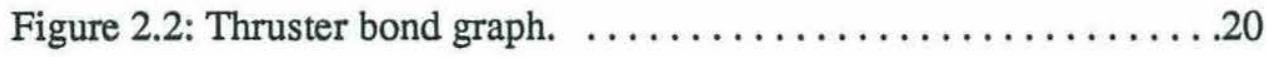

Figure 2.3: Normalized step response of the thruster model. . . . . . . .21

Figure 3.1: Schematic representation of the test setup used to conduct

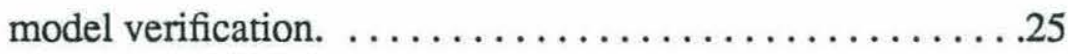

Figure 3.2: Linear relationship between thrust and $\omega|\omega| \ldots \ldots \ldots 26$

Figure 3.3: Linear relationship between $\omega|\omega|$ and input control voltage,

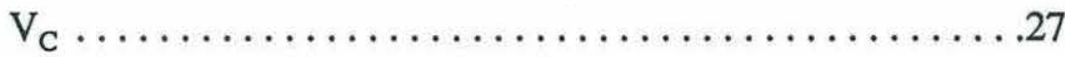

Figure 3.4: Linear relationship between $v_{\mathrm{f}}$ and $\omega \ldots \ldots \ldots \ldots \ldots 28$

Figure 3.5: Comparison of simulation and recorded data of thruster response to a random input. $\ldots \ldots \ldots \ldots \ldots \ldots \ldots \ldots \ldots$

Figure 3.6: Comparison of derived and recorded data of thruster response to a random input. $\ldots \ldots \ldots \ldots \ldots \ldots \ldots \ldots \ldots \ldots \ldots \ldots \ldots$

Figure 4.1: Example of $1 \mathrm{~V}$ input step response with a corresponding first-

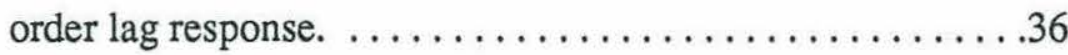

Figure 4.2: Improvement in the thruster step response with lead

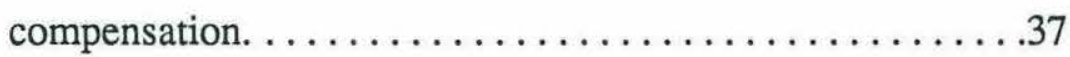

Figure 4.3: Functional form of $\lambda_{v}$ matched to empirical data. . . . . . 39

Figure 5.1: System setup for hybrid simulation $\ldots \ldots \ldots \ldots \ldots \ldots$ 
Figure 5.2: Effect of thruster motor saturation without thruster dynamic compensation. .........................54

Figure 5.3: Effect of thruster dynamics without thruster dynamic compensation at low thrust. $\ldots \ldots \ldots \ldots \ldots \ldots \ldots .54$

Figure 5.4: Improved vehicle tracking performance obtained with lead compensation. ............................ 56

Figure 5.5: Reduced effectiveness of lead compensation at high thrust. .57 Figure 5.6: Reduced effectiveness of lead compensation at low thrust. . .57 Figure 5.7: Root locus plots demonstrating the results of operating away from the point of lead compensator design and system linearization points. $\ldots \ldots \ldots \ldots \ldots \ldots \ldots \ldots \ldots . \ldots 5$

Figure 5.8: Vehicle tracking performance with "pole" cancellation thruster controller. .............................59

Figure 5.9: Degraded "pole" cancellation thruster control with obstruction in thruster duct outlet. . . . . . . . . . . . . . . . . . 60

Figure 5.10: Vehicle tracking performance with adaptive sliding thruster controller. ............................

Figure 5.11: Thruster model parameter, c convergence. .........66

Figure 5.12: Thruster model parameter, $h$ convergence. . .........62

Figure 5.13: Adaptive sliding thruster control with obstruction in the thruster duct outlet. $\ldots \ldots \ldots \ldots \ldots \ldots \ldots \ldots \ldots \ldots 63$

Figure 6.1: Relationship between torque, static thrust and input command.

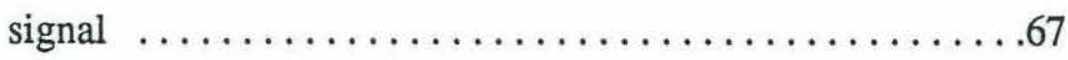

Figure 6.2: Schematic representation of the propeller speed sensor configuration. . . . . . . . . . . . . . . . . . . .69 
Figure 6.3: Schematic representation of the complete thruster test facility. .................... 


\section{Chapter 1}

\section{INTRODUCTION}

\subsection{Motivation}

The Deep Submergence Laboratory at the Woods Hole Oceanographic Institution has developed and deployed a deep ocean remotely operated vehicle known as JASON. JASON is a highly maneuverable vehicle capable of operating in automatic and operator interactive position and trajectory following modes. High resolution, high update rate navigation information for the control of JASON is obtained from a high frequency acoustic system known as SHARPS (Sonic High Accuracy Ranging and Positioning System) [1]. JASON is also equipped with a manipulator specifically designed for precise position and force control. The JASON vehicle is shown in figure 1.1 .

With the available high resolution (approximately $1 \mathrm{~cm}$ ) position information, JASON exhibits an approximately $10 \mathrm{~cm}$ diameter watch circle in a hover mode. This limit cycle behavior is attributed to a combination of poor thruster performance at low amplitudes and time delays in the computer control system. [1]. For most tasks and in comparison to comparable systems, this is excellent performance. However, this position control accuracy is not adequate to provide a stable platform for manipulator tasks or ultimately to allow coordinated vehicle/manipulator motion. The limit cycle during hover 


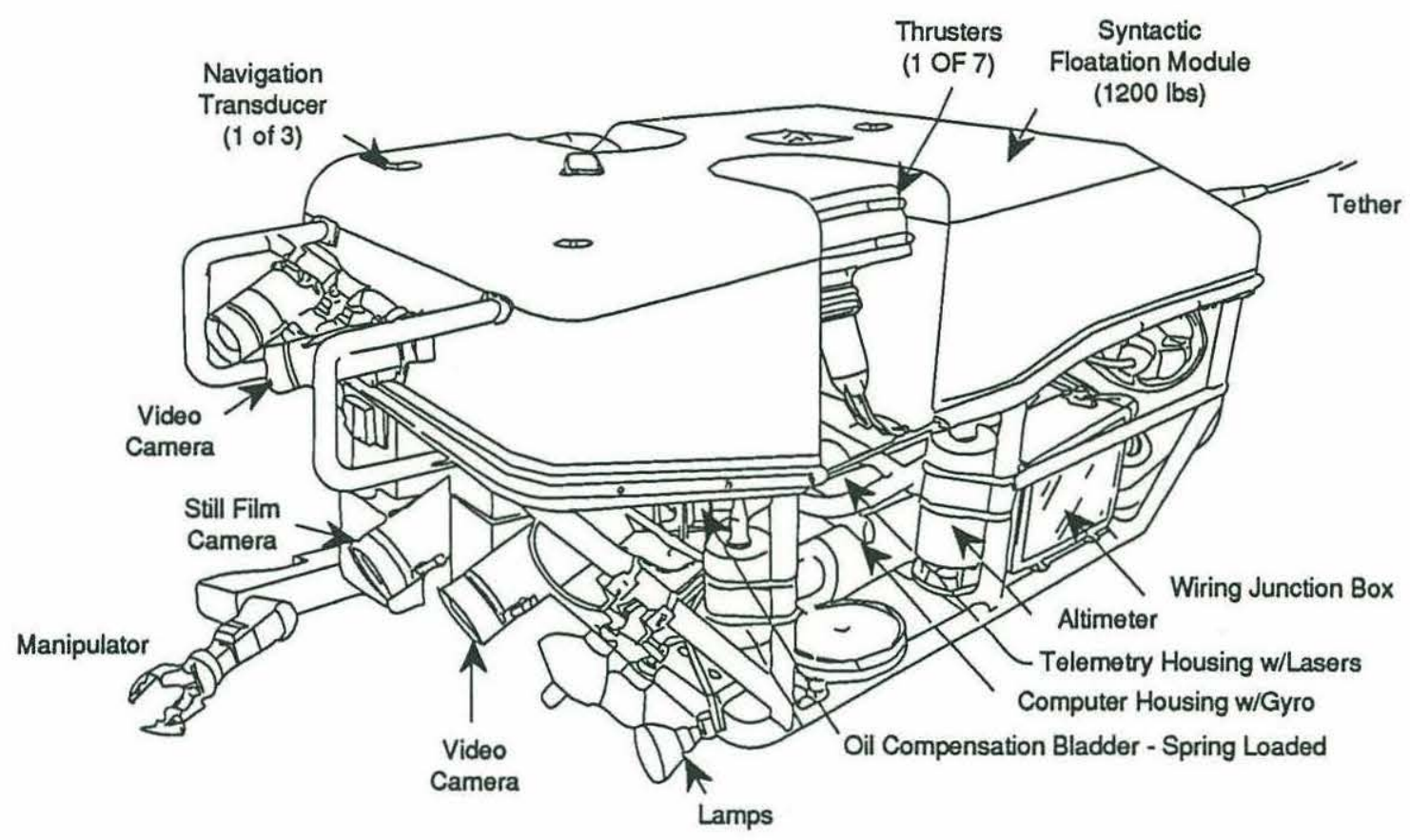

Figure 1.1: The remotely operated vehicle JASON.

operations is the most significant shortcoming of the current implementation of the ROV JASON [1]. The incorporation of thruster dynamics in the control of JASON has the potential to eliminate this shortcoming and significantly improve performance.

In the design and implementation of control systems, the dynamics associated with the actuators are frequently neglected on the basis of their characteristically high bandwidth nature. The assumption that the actuator dynamics are to be ignored in the control system implementation is an important consideration in the proper selection of actuators. The actuator typically utilized on underwater vehicles, including JASON, is the ducted propeller, referred to as a thruster. The dynamics of the thruster are nonlinear and characteristically of a low bandwidth nature. These characteristics can be dominant in the control of an underwater vehicle when low control action is required, as in a hover mode. 
This research work was motivated by the need to develop a position control system for underwater vehicles capable of taking full advantage of the available high bandwidth position information. To this end, it is considered imperative that an underwater vehicle which relies on thrusters to deliver control action should compensate in its position control for the dominant actuator dynamics.

\subsection{Research Objectives}

To control a dynamic system in some optimum sense, one must have an understanding of the important physical phenomena affecting the dynamics and a model which captures those phenomena. Therefore, the first research objective is the development of a model of the thruster dynamics. The model should be physically-based as an improvement in control through an increased understanding of the actuator dynamics is the desired outcome. The model should be developed from energy considerations to permit coupling between energy domains including the torque source (electric motor), propeller and ambient fluid. The dynamics should be characterized by practically observable phenomena of the existing thruster configuration. For example, direct measurement of rotor angular velocity may be practical while rotor torque or the fluid flow field are not.

With any model, there are simplifications of the true physical processes with resulting model uncertainty. To verify the simplifications are valid over the range of interest and to quantify the model parameters, actual thruster dynamics should be observed. Hence, the second research objective is the design, construction, and operation of a thruster test facility which permits full observation and recording of the thruster dynamic response. The test facility should verify the viability, and accuracy of 
measurement of the thruster dynamic state and be flexible enough to permit the implementation and testing of various control schemes.

With the model developed and verified, control system design may proceed. The third and central research objective is the development of a control system capable of compensating for the nonlinear thruster dynamics which can be integrated into a vehicle position control scheme.

Accomplishment of the above objectives should as well provide insight into implementation issues associated with incorporating thruster dynamics in the current vehicle configuration as well as thruster configuration changes which might reduce the dynamic effects.

\subsection{Outline of Thesis}

Chapter 2 presents the lumped parameter model development of the thruster using energy-based physical system modeling of an open system. Chapter 3 contains the experimental verification of the developed thruster model demonstrating it correctly models the thruster dynamic response. System identification techniques are utilized to determine the model parameters which best match the actual dynamics.

Chapter 4 addresses the design of thruster controllers which can compensate for the thruster dynamics. A lead compensation controller is designed which assumes the thruster dynamics are characterized by a linear first-order lag. A "pole" cancellation controller is designed which adjusts for the apparent changes in the thruster lag characteristics with the amplitude of the applied torque. The form of the "pole" cancellation controller is shown to be model-based. An adaptive sliding mode controller is designed which takes advantage of the thruster dynamic model as well as feedback of the thruster dynamic state. 
Chapter 5 contains an evaluation of each of the controller designs in the context of vehicle tracking performance using a hybrid simulation technique.

Chapter 6 provides a technical description and configuration of the test facility developed to support the model verification and controller evaluation.

Chapter 7 summarizes the results of the thesis and offers recommendations for additional research. 


\section{Chapter 2}

\section{THRUSTER DYNAMICS}

A typical thruster configuration consists of a propeller surrounded by a static shroud, coupled mechanically to an electric motor. The electric motor is controlled by a servoamplifier with control voltage input and a corresponding current output. The output current is converted to a torque in the electric motor which is transferred to the water by the propeller thereby creating a thrust. It is generally assumed that all the aforementioned conversions are linear and time-invariant resulting in a direct correspondence between the input control voltage and output thrust. This simplification ignores any time delays, deadband, or hysteresis effects but more importantly it ignores the thruster dynamics.

The model development that follows will concentrate on the thruster dynamics and assumes the electric motor operates as a torque source. [2] demonstrated the applicability of bond graph/energy-based physical system modeling to open systems and introduced a methodology for their treatment. The techniques illustrated in [2] will be used here to develop the dynamic model of a thruster.

\subsection{Lumped Parameter Model Development}

A typical thruster is schematically represented in figure 2.1 and consists of a static shroud and a propeller driven by a torque source $(\tau)$ at angular velocity $(\omega)$. The 


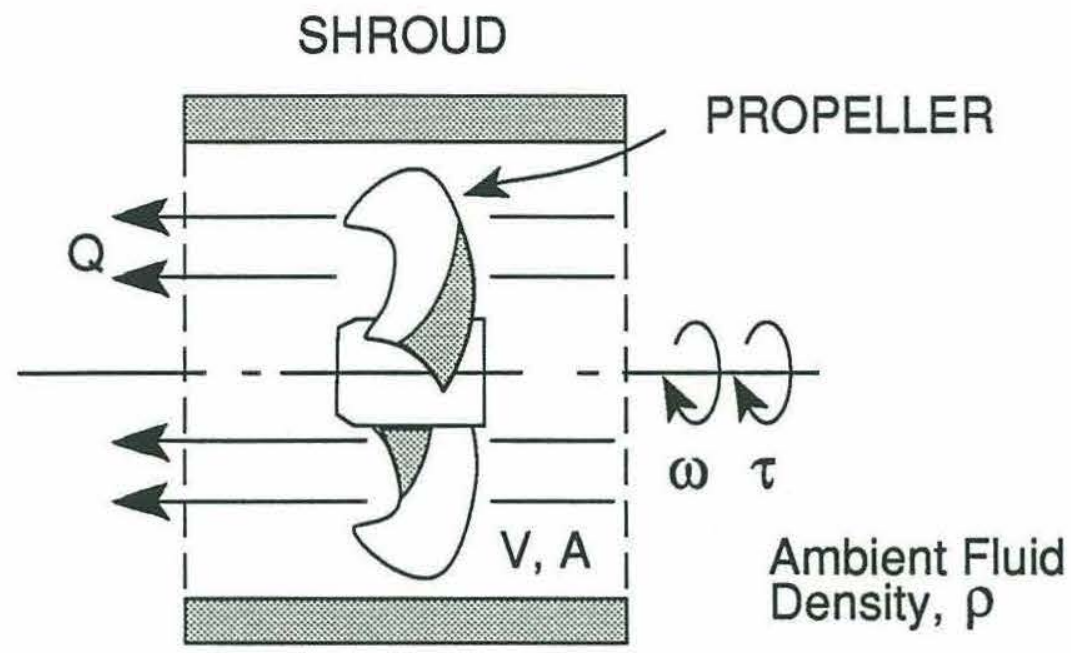

Figure 2.1: Typical thruster schematic.

thruster shroud has a cross-sectional area (A) and encloses a volume (V). The ambient fluid has density $(\rho)$ and a volumetric flowrate within the thruster (Q).

The model development is simplified by the following assumptions:

- The energy stored is solely due to the kinetic energy of the fluid.

- The kinetic energy of the ambient fluid is negligible.

- Friction losses are negligible.

- Ambient fluid is incompressible.

- Fluid flow at the thruster intake and exhaust is parallel, one dimensional and at ambient pressure.

- Gravity effects are negligible.

The kinetic coenergy, $\mathrm{T}^{*}$ of the fluid in the thruster can be expressed as a state function of the volumetric flowrate, Q.

$$
\mathrm{T}^{*}(\mathrm{Q})=\frac{1}{2} \rho \mathrm{V}\left[\frac{\mathrm{Q}}{\mathrm{A}}\right]^{2}
$$


A generalized momentum, $\Gamma$ can be defined as,

$$
\Gamma=\frac{d \Gamma^{*}}{d Q}=\rho V \frac{Q}{\mathrm{~A}^{2}}
$$

The above relation is that of an inertia (momentum related by a static constitutive law to the flow [3]) in bond graph nomenclature with effort variable, $\Gamma$ and flow variable, Q. $\Gamma$ has units of momentum/area and is referred to as the pressure momentum. Since the energy relations are linear, the coenergy and energy have equal magnitudes [4], and the kinetic energy, $\mathrm{T}$ can be expressed as a state function of the pressure momentum, $\Gamma$,

$$
\mathrm{T}(\Gamma)=\frac{\mathrm{A}^{2}}{2 \rho \mathrm{V}} \Gamma^{2}
$$

A power balance yields the following pressure momentum relation,

$$
\frac{d \mathrm{~T}}{d t}=\frac{\mathrm{A}^{2}}{\rho \mathrm{V}} \Gamma \Gamma=\omega \tau-\mathrm{KQ}
$$

where,

$\omega \tau$ represents the power input from the thruster propeller.

$\mathrm{K}$ represents the exiting kinetic energy per volume.

$\Gamma \quad$ represents the time rate of change of the pressure momentum.

The exiting kinetic energy per volume, $\mathrm{K}$ can be expressed as,

$$
\mathrm{K}=\frac{\mathrm{A}^{2} \Gamma^{2}}{2 \rho \mathrm{V}^{2}}=\frac{\gamma^{2}}{2 \rho}
$$

where,

$\gamma \equiv \frac{\mathrm{A} \Gamma}{\mathrm{V}}$ is the fluid momentum per volume within the thruster. 
The thruster and ambient fluid are coupled through the convected linear momentum, which is equal to the thrust developed,

$$
\text { Thrust }=\gamma \mathrm{Q}
$$

Assuming the propeller does not cavitate, the volumetric flowrate can be related to the thruster/propeller characteristics and angular velocity, $\omega$. The difference between the theoretical and actual advance per revolution of a propeller is referred to as the slip and is typically expressed as a ratio, $\sigma$ as follows,

$$
\sigma=\frac{\omega \mathrm{pA}-\mathrm{Q}}{\omega \mathrm{pA}}
$$

where,

p represents the axial distance traveled by the propeller blades with each revolution and is referred to as the pitch.

It follows from the equation above that $\mathrm{Q}(\omega)$ can be expressed as,

$$
\mathrm{Q}=\eta \mathrm{pA} \omega
$$

where,

$\eta \equiv 1-\sigma$ and is referred to as the propeller efficiency.

From equations 2.1.1, 2.1.2, 2.1.3 and 2.1.4 the following thruster dynamic state and output equations are formed,

$$
\begin{aligned}
& \Gamma=\frac{\tau}{\eta \mathrm{pA}}-\mathrm{K} \\
& \text { Thrust }=\gamma \mathrm{Q}
\end{aligned}
$$

The thruster dynamic state and output equations can be represented topographically in the bond graph of figure 2.2. 


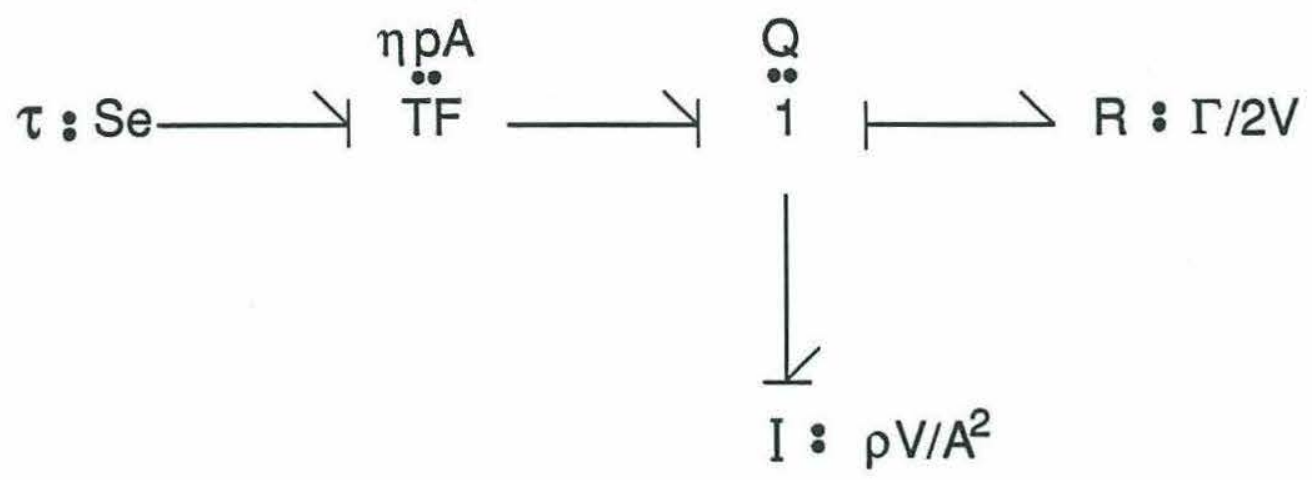

Figure 2.2: Thruster bond graph.

If we assume that the propeller efficiency $(\eta)$, pitch (p), and duct area (A) are constant, the thruster dynamic state and output equations may be expressed with the propeller angular velocity, $\omega$ as the thruster dynamic state variable,

$$
\begin{gathered}
\omega=\frac{\tau}{\eta^{2} \mathrm{p}^{2}} \rho \mathrm{V}-\frac{\eta p \mathrm{~A}}{2 \mathrm{~V}} \omega|\omega| \\
\text { Thrust }=\mathrm{A} \rho \eta^{2} \mathrm{p}^{2} \omega|\omega|
\end{gathered}
$$

The normalized step response of the thruster model to torques of $2,1 / 3$ and $1 / 4$ $\mathrm{Nm}$ is presented in figure 2.3, graphically emphasizing the nonlinear dynamics. The thruster presents a more complex control problem than the linear first-order lag associated with most actuators. The thruster time response performance actually degrades as the magnitude of the input decreases. This dynamic behavior, when coupled with other dynamic nonidealities such as a pure delay could result in the limit cycle behavior associated with underwater vehicle hover control. 


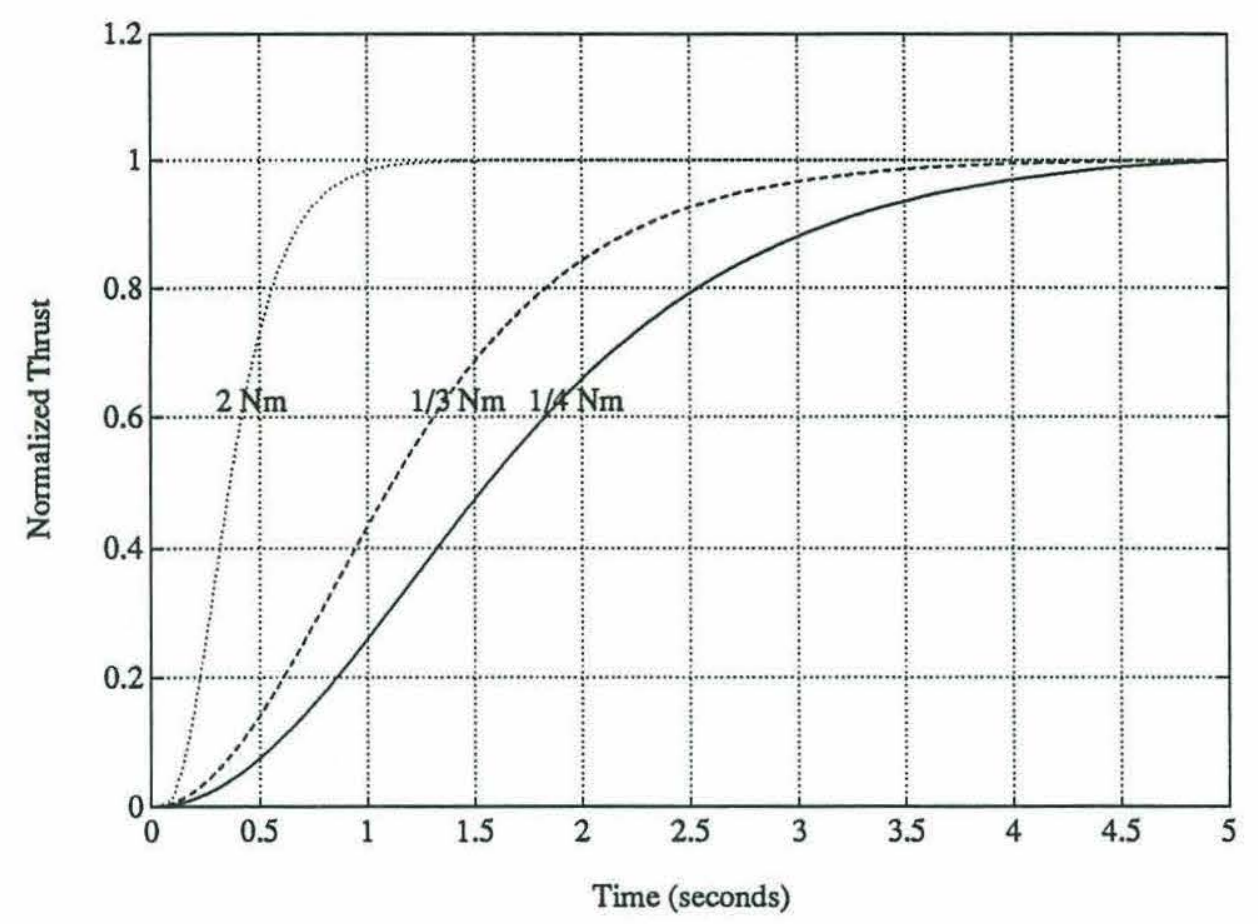

Figure 2.3: Normalized step response of the thruster model.

\subsection{Summary}

Using an energy-based, physical system approach, a lumped parameter dynamic model of the thruster was developed. With the propeller angular velocity, $\omega$ as the thruster dynamic state, the model and the output equation take the form,

$$
\begin{aligned}
& \omega=\beta \tau-\alpha \omega|\omega| \\
& \text { Thrust }=C_{t} \omega|\omega|
\end{aligned}
$$

where,

$\tau$ is the input torque.

$\alpha$ and $\beta$ are constant model parameters.

$\mathrm{C}_{\mathrm{t}}$ is a proportionality constant. 
This model will be used in subsequent chapters as a dynamic structure for determining the constant model parameters and ultimately in the design of controller systems to compensate for the thruster dynamics. 


\section{Chapter 3}

\section{THRUSTER MODEL VERIFICATION}

The goal of the model verification is to ensure the developed thruster model structure is correct, to determine the numerical value of the model parameters which best match the thruster being tested, and to confirm that these numerical values are consistent with the measurable physical characteristics of the thruster. The model is verified first using the common static propeller relations, then a dynamic verification is performed to yield numerical values for the full dynamic model.

\subsection{Experimental Setup}

The model verification required the measurement and logging of the following,

Input Thruster motor control voltage.

Dynamic State Propeller angular velocity.

Fluid velocity at the thruster duct outlet.

Output Thrust.

Data acquisition and control was provided by a personal computer. The thruster input was supplied by an analog output controller. Propeller angular velocity was sensed by an electro-optic device mounted in the thruster stator hub and processed 
through a counter/timer controller. The fluid velocity at the outlet of the thruster duct was measured by an analog electromagnetic current meter. Thrust was sensed by a load cell located between the thruster motor and the supporting test stand and processed through a strain gage signal conditioner. The test setup used to conduct the model verification is presented schematically in figure 3.1 and a more detailed explanation of the full experimental setup can be found in chapter 6 .

\subsection{Static Relations}

A propeller and hence a thruster are typically treated as static devices, with a constant gain relating the output thrust to the input torque. For the thruster configuration tested, the motor is torque-controlled, hence the input control voltage is equivalent to the input torque and will be used in place of torque in subsequent model descriptions. Assuming steady-state conditions, this relation is confirmed in the model description. Recall the thruster model and output equations of chapter 2,

$$
\begin{aligned}
& \omega=\beta \tau-\alpha \omega|\omega| \\
& \text { Thrust }=C_{t} \omega|\omega|
\end{aligned}
$$

substituting into the thruster model assuming steady-state $(\omega \equiv 0)$,

$$
\begin{gathered}
\mathrm{V}_{\mathrm{C}}=\frac{\alpha}{\beta} \omega|\omega| \\
\text { Thrust }=\mathrm{C}_{\mathrm{t}} \frac{\beta}{\alpha} \mathrm{V}_{\mathrm{C}}
\end{gathered}
$$

where,

$\mathrm{C}_{\mathrm{t}}$ is a proportionality constant.

$\omega$ is the propeller angular velocity.

$\alpha$ and $\beta$ are model parameters.

$\mathrm{V}_{\mathrm{C}}$ is the input control voltage (substituted for torque). 


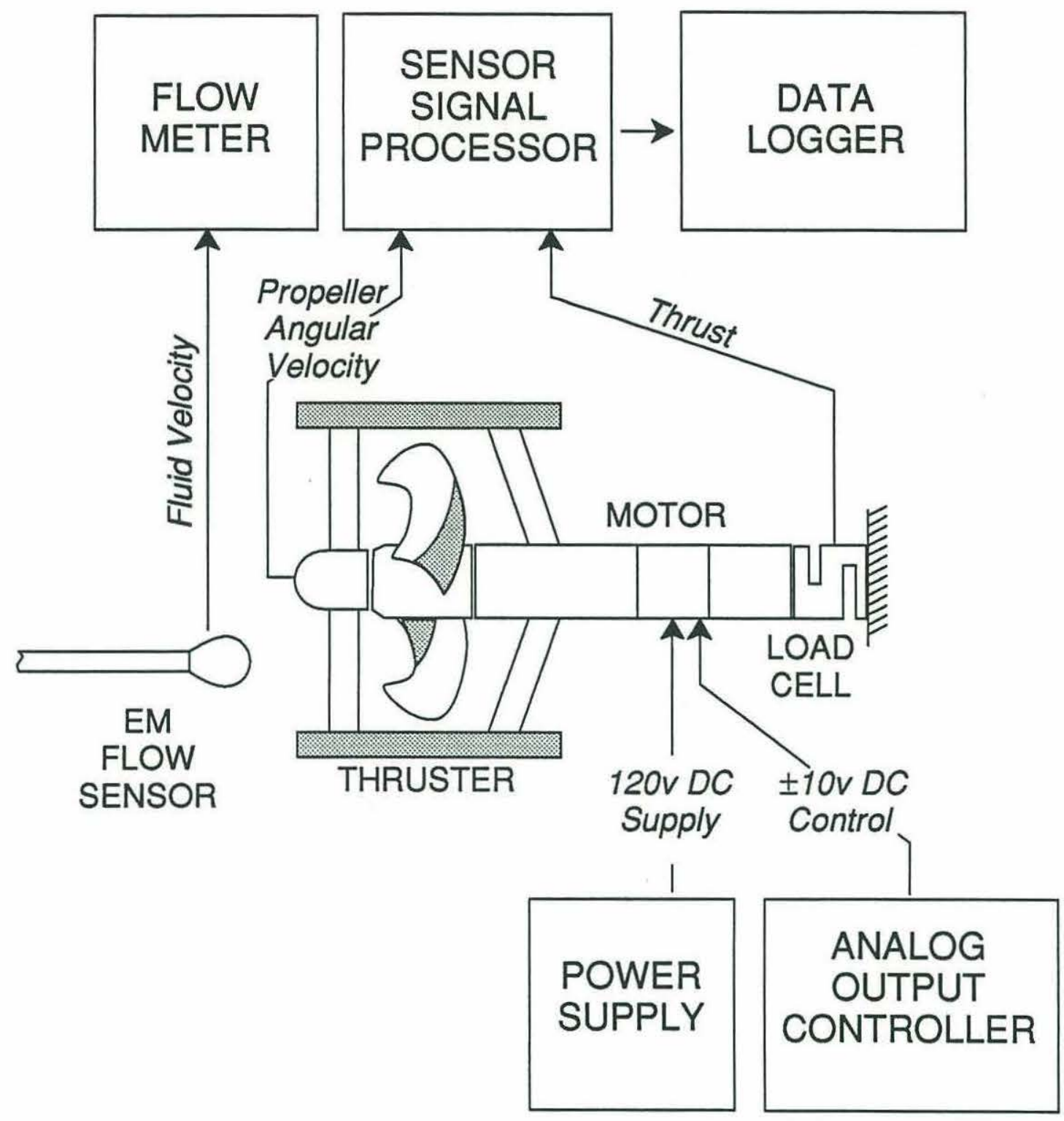

Figure 3.1: Schematic representation of the test setup used to conduct model verification.

The above static relations were verified to be valid and numerical values determined for the proportionality constants using steady-state measurements of the thruster's response to a step input. 


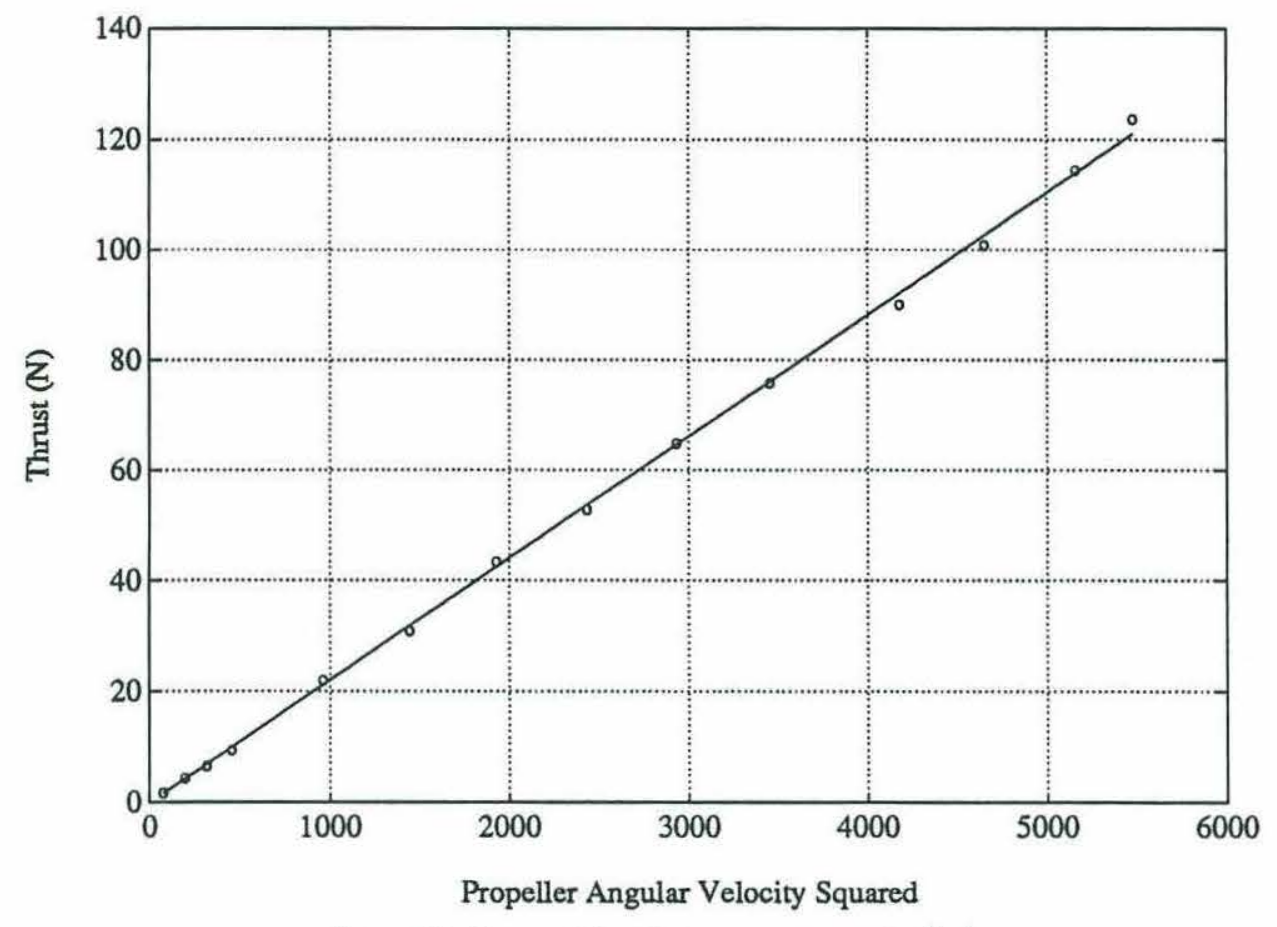

Figure 3.2: Linear relationship between thrust and $\omega|\omega|$

Figure 3.2 illustrates the relationship between thrust and $\omega|\omega|$. The modeled linear relationship is satisfied with a least-square fit yielding a numerical value, $\mathrm{C}_{\mathrm{t}}=$ $.0221 \mathrm{Ns}^{2}$.

Figure 3.3 illustrates the relationship between $\omega|\omega|$ and the input control voltage, $\mathrm{V}_{\mathrm{C}}$. The modeled linear relationship is satisfied with a least-square fit yielding a numerical value, $\frac{\beta}{\alpha}=1037$ volts $^{-1} \mathrm{~s}^{-2}$.

Combining the above results yields a numerical value for the thruster static input-output relation,

$$
\text { Thrust }=22.9 \mathrm{~V}_{\mathrm{C}}
$$

In the thruster model and in the static relations above, it is assumed that there is close coupling, hence a linear relationship between the volumetric flow rate and the propeller angular velocity allowing $\omega$ to be used as the thruster dynamic state. The linear 


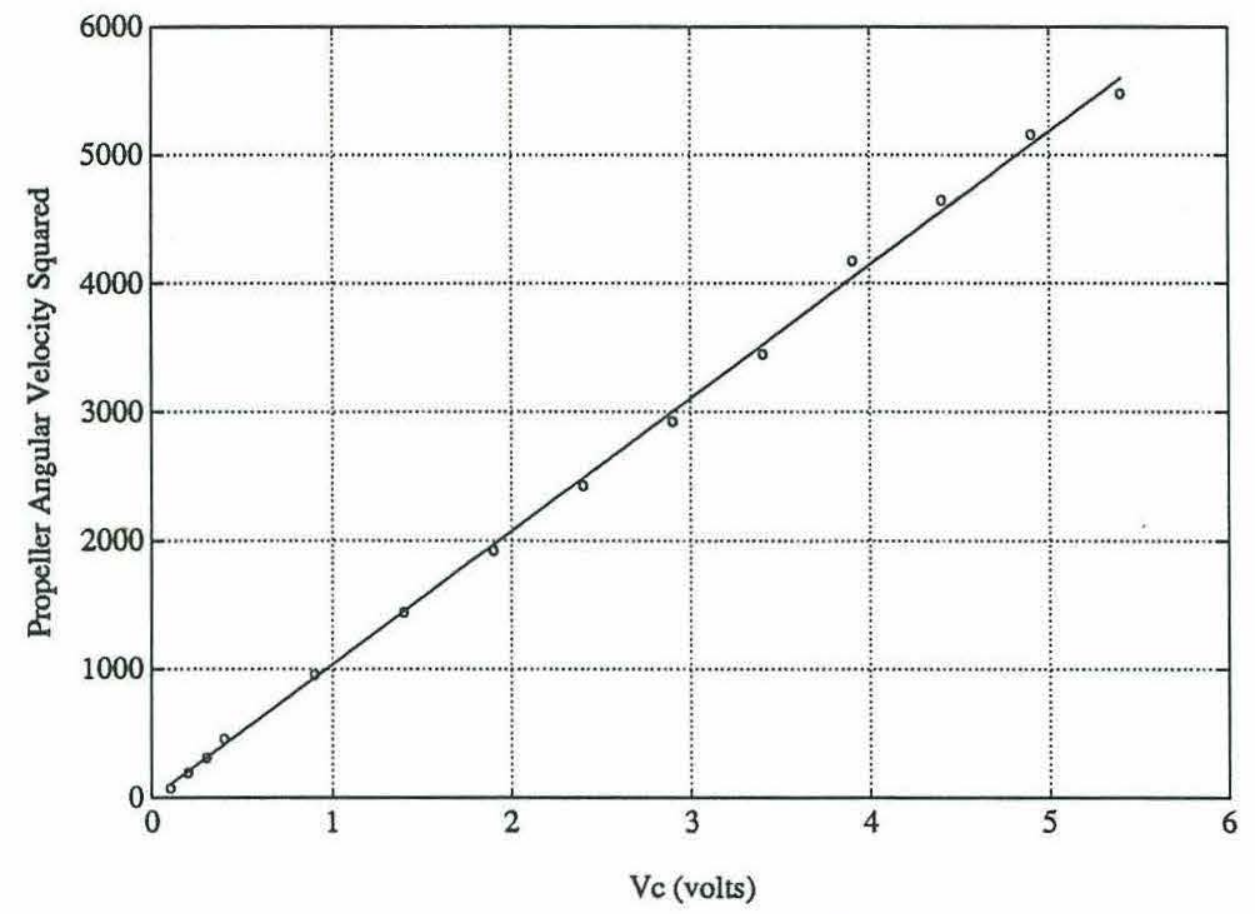

Figure 3.3: Linear relationship between $\omega|\omega|$ and input control voltage, $V_{C}$

relationship is verified using data from the flow measurements at the outlet of the thruster duct. Recall from the thruster model development,

$$
\frac{\mathrm{Q}}{\mathrm{A}}=\eta \mathrm{p} \omega=v_{\mathrm{f}}
$$

where,

$v_{\mathrm{f}}$ is the fluid velocity at the thruster outlet.

$\eta$ is the propeller efficiency.

$\mathrm{p}$ is the propeller pitch.

A is the thruster cross-sectional area.

Figure 3.4 illustrates the approximately linear relationship between the fluid velocity at the thruster outlet and the propeller angular velocity, $\omega$. The data presented indicates propeller efficiency may be a weak function of $\omega$. No functional dependence is 


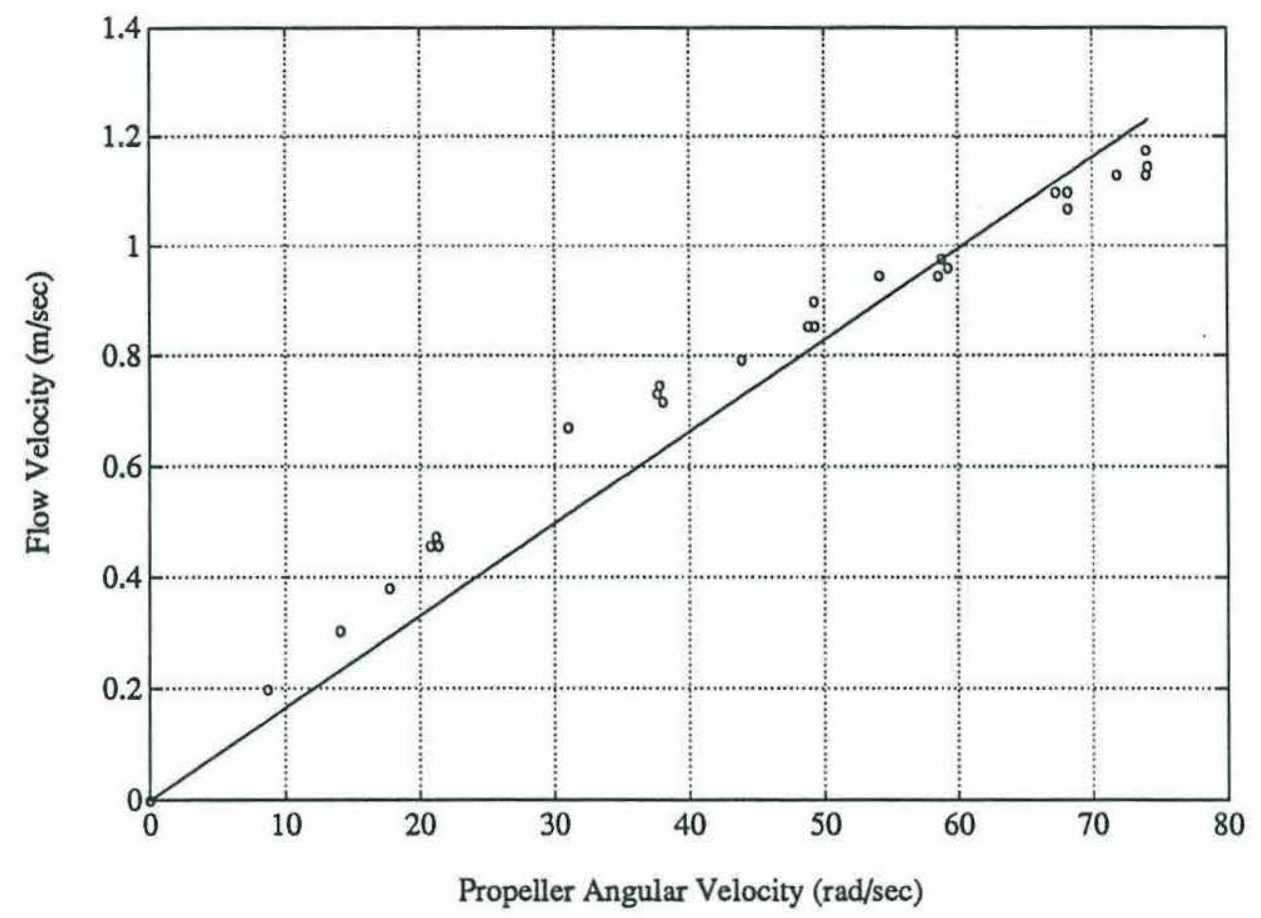

Figure 3.4: Linear relationship between $v_{\mathrm{f}}$ and $\omega$

evident however in the relationship between thrust and $\omega|\omega|$ either in the static case presented or in the dynamic verification of the next section. The electromagnetic current sensor used to measure the fluid velocity at the thruster outlet is sensitive to orientation and the distance from the duct. This sensitivity may have degraded accurate measurements at the higher velocities.

\subsection{Dynamic Relations}

The static relations developed from the thruster step response are not sufficient to fully specify the thruster model parameters. The complete dynamic relations were determined using the thruster's response to a random input generated by filtering a normal distribution noise signal with a $1 \mathrm{~Hz}$ cutoff frequency. 
For the dynamic verification, some changes to the experimental setup were required. The propeller angular velocity alone was used as the measure of the thruster dynamic state as the analog flow meter does not have sufficient dynamic range. The direct output of the speed sensor is a series of pulses, each of which represents an arc length of propeller travel. Therefore, the actual output of the sensor is propeller "position". This signal was recorded directly and processed off line to provide the thruster state measurement and its derivative. The propeller "position" signal is noisefree and hence can be accurately differentiated twice to obtain the thruster state derivative, $\omega$. Smoothing of the angular velocity was required however to remove digital sampling effects. As in the static tests, the thrust was sensed by the load cell mounted between the thruster motor and the supporting test stand.

The model form used in the dynamic verification was,

$$
\omega=\beta V_{C}-\alpha \omega|\omega|
$$

The random control signal was input to the thruster with propeller angular "position" and thrust recorded. As described previously, the propeller angular position was then differentiated and smoothed to generate the thruster dynamic state, $\omega$ and its derivative, $\omega$. A least-squares fit of the data to the model structure yielded the numerical parameter values,

$$
\begin{gathered}
\alpha=0.0372 \text { dimensionless } \\
\beta=41.87 \text { volts }^{-1} \mathrm{~s}^{-2} \\
\frac{\beta}{\alpha}=1126 \text { volts }^{-1} \mathrm{~s}^{-2}
\end{gathered}
$$

It should be noted immediately that these values match well with the static relations, where $\frac{\beta}{\alpha}=1037$ volts $^{-1} \mathrm{~s}^{-2}$. 


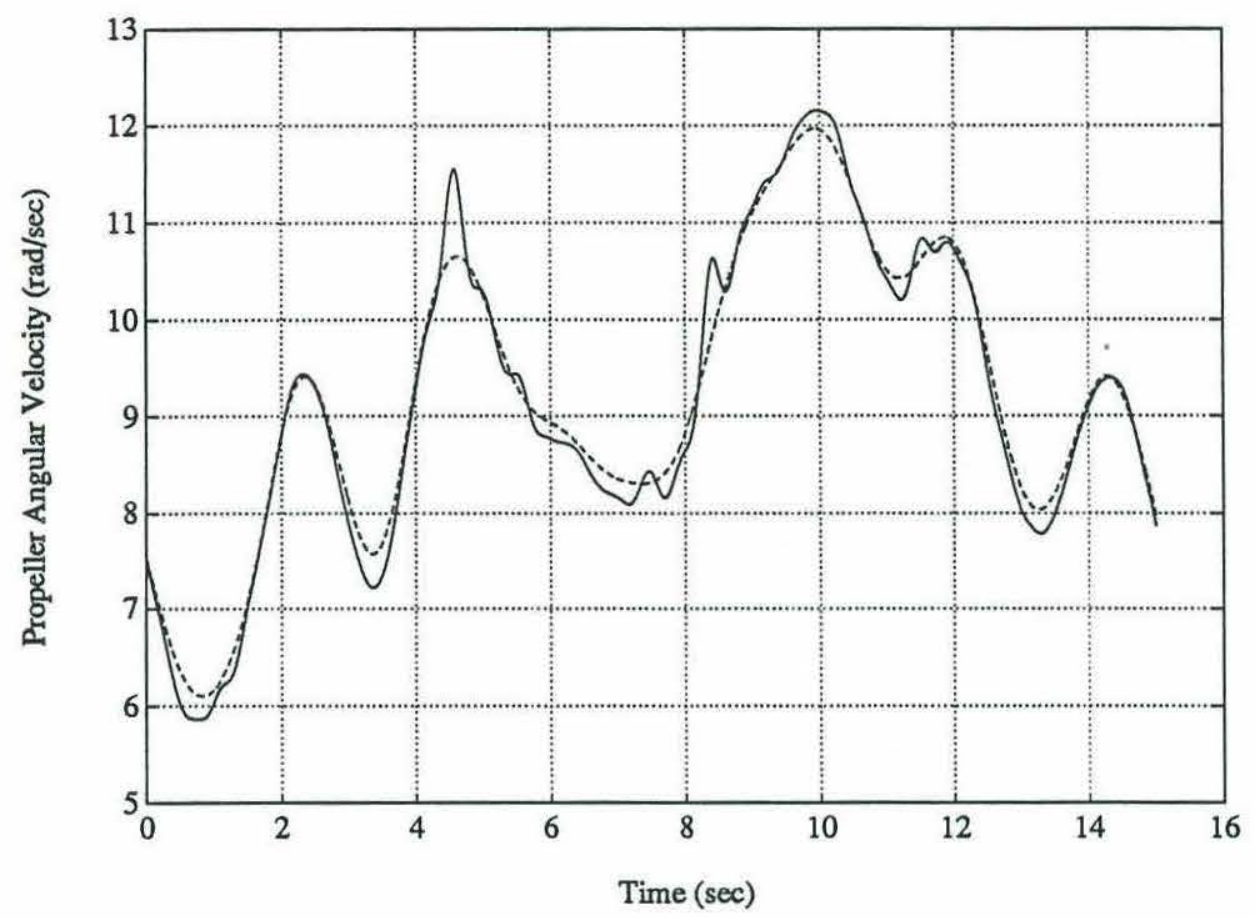

Figure 3.5: Comparison of simulation and recorded data of thruster response to a random input.

[- actual response - - simulated response]

These parameters were utilized in a simulation of the thruster response to the same input. The results of the simulation are compared to the recorded data graphically in figure 3.5. The RMS error is $1.8 \mathrm{rad} \mathrm{s}^{-1}$.

In determining the static relations, it was assumed that,

$$
\text { Thrust }=\mathrm{C}_{\mathrm{t}} \omega|\omega|
$$

where,

$\mathrm{C}_{\mathrm{t}}$ is a proportionality constant, determined from the static relations to be $0.0221 \mathrm{Ns}^{2}$.

Similarly, the proportionality constant, $\mathrm{C}_{\mathrm{t}}$ may be determined from the dynamic response. A least squares-fit of the dynamic verification data yielded $\mathrm{C}_{\mathrm{t}}=.0189 \mathrm{Ns}^{2}$, which compares well with the value obtained from the static relation. Thrust as derived 


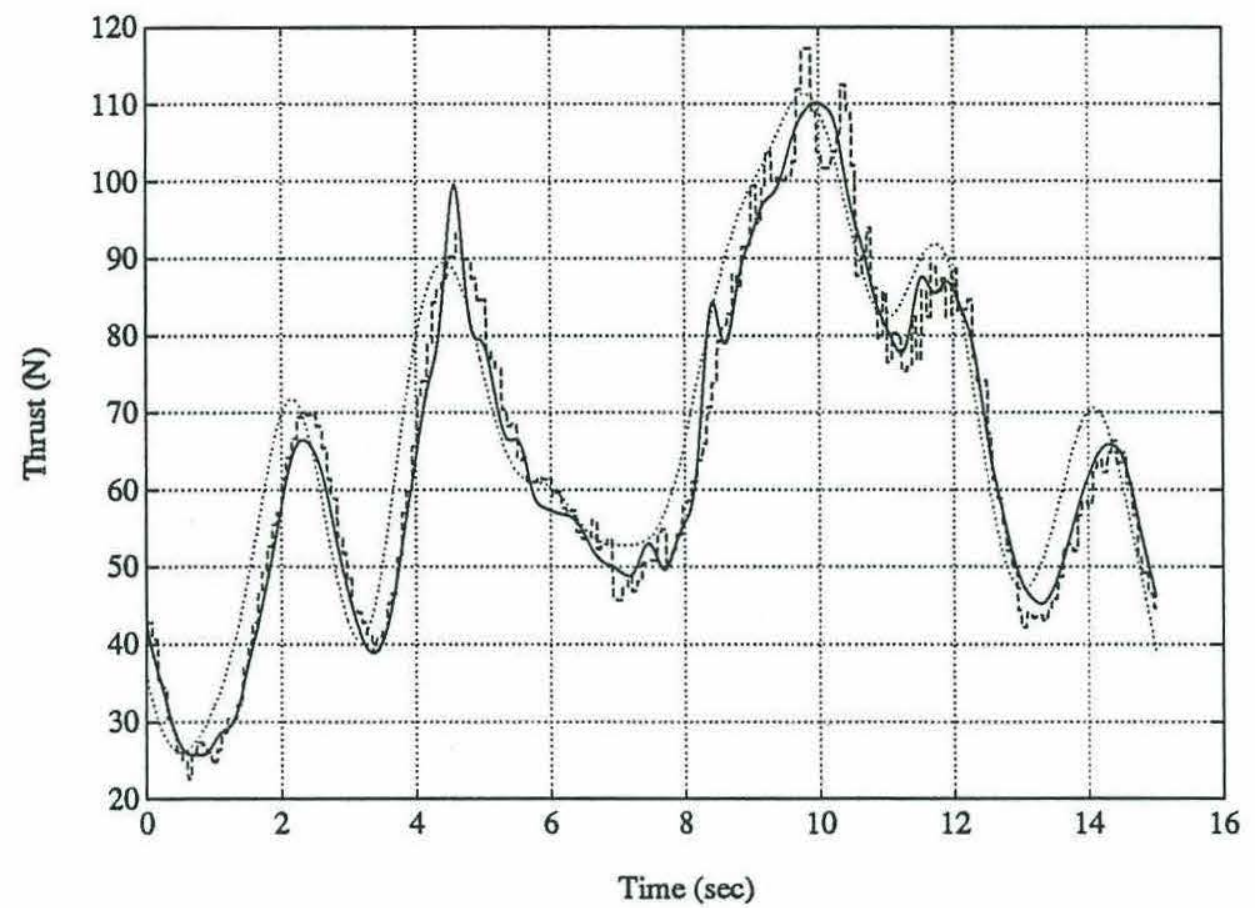

Figure 3.6: Comparison of derived and recorded data of thruster response to a random input.

$\left[-\mathrm{C}_{\mathrm{t}} \omega|\omega|\right.$ response - - recorded thrust $\cdots$ command input $]$

from the measured propeller angular velocity squared and the proportionality constant is compared graphically to the measured thrust in figure 3.6. Plotted with the thrust is the commanded input, emphasizing the phase lag.

\subsection{Parameter Comparisons}

To confirm that the numerical values obtained are consistent with the measurable physical characteristics, we return to the following form of the thruster dynamic state and output equations of chapter 2, 


$$
\begin{aligned}
& \omega=\frac{\tau}{\eta^{2} \mathrm{p}^{2} \rho \mathrm{V}}-\frac{\eta \mathrm{pA}}{2 \mathrm{~V}} \omega|\omega| \\
& \text { Thrust }=\mathrm{A} \rho \eta^{2} \mathrm{p}^{2} \omega|\omega|
\end{aligned}
$$

Many of the terms which appear in the above relations are physical constants or known thruster geometric characteristics. These terms and their numerical values are listed below,

p, propeller pitch $0.101 \mathrm{~m}$
A, thruster duct area $0.0527 \mathrm{~m}^{2}$
$\rho$, fluid density $1000 \mathrm{~kg} / \mathrm{m}^{3}$

From the thruster motor specifications, the applied motor torque as a function of the input control voltage is,

$$
\tau=0.339 \mathrm{~V}_{\mathrm{C}} \mathrm{Nm} / \mathrm{volt}
$$

The thruster model development assumed the involved fluid volume, $\mathrm{V}$ was equal to the volume of fluid enclosed by the thruster duct, however the actual involved volume cannot be determined exactly from thruster geometric characteristics. Likewise, the propeller efficiency, $\eta$ cannot be determined from physical constants or geometric considerations.

Equating the coefficients of equations 3.4.1 and 3.4.2 to the numerical values determined in the dynamic verification yields numerical values for the unknown constants, $\mathrm{V}$ and $\eta$,

$$
\mathrm{V}=0.016 \mathrm{~m}^{3}
$$

which is equivalent to the volume enclosed by a duct of the same cross-sectional area but twice the length as the thruster tested. 


$$
\eta=22 \%
$$

which is well within the range of measured efficiencies for propellers of this type.

\subsection{Summary}

The thruster dynamic model developed accurately describes the actual thruster dynamics over the range of interest.

The structure of the thruster dynamic model is verified to be correct using static and dynamic measurement of the actual thruster response. Numerical values of the model parameters were obtained and compared using static and dynamic relations. Comparison of the parameter numerical values with known physical and geometric relations verifies that in addition to the proper structure, the thruster dynamic model represents an accurate physical interpretation.

The model parameter numerical values used in the subsequent controller design and evaluation are,

$$
\begin{gathered}
\alpha=0.0372 \\
\beta=41.87 \text { volts }^{-1} \mathrm{~s}^{-2} \\
\mathrm{C}_{\mathrm{t}}=0.0189 \mathrm{Ns}^{2}
\end{gathered}
$$




\section{Chapter 4}

\section{CONTROLLER DESIGN}

Having derived and verified a dynamic model of the thruster, we turn our attention to the controller design. The approach presented has at its foundation, simplicity and practicality. Initial designs are developed assuming there is no thruster dynamic state measurement. These simple controller designs are based on observations of the thruster step response only, while the dynamic model provides a means of qualitatively evaluating the validity of the controller structure. These controllers demonstrate what improvements to thruster dynamic performance can be expected without investing in additional sensors and provide a benchmark for the evaluation of a more complete modelbased controller which requires the measurement of the thruster dynamic state.

Assuming a measurement of the thruster dynamic state is available, a modelbased controller is developed using the concept of a sliding surface. Adaptive control techniques are utilized to insure the controller will be robust to model uncertainty and disturbances.

\subsection{Lead Compensation}

A qualitative examination of the thruster step response in the time domain reveals that the thruster might be modeled as a linear first-order system with a time 
constant dependent on the magnitude of the input control action signal. Despite the contradictory nature of this observation (a time constant that is not constant), the use of a simple linear model permits the application of familiar control system design techniques. An example of the thruster step response with a $1 \mathrm{~V}$ control input and a corresponding first-order lag response is shown in figure 4.1.

With the thruster modeled as a first-order lag, lead compensation represents a simple method of improving the transient response without requiring additional actuator (thruster) sensors. The advantage of lead compensation is in the implementation. An analog lead network can easily be placed directly in line with the control signal with accompanying amplification of the commanded signal to correct for the attenuation of the network. Lead compensation in this simple form cannot however adjust to the apparent changes in the time constant.

The time constant selected as representative of the dynamics to be compensated is that observed at low control signals. From figure 4.1, we observe that a 0.75 second time constant matches the observed step response at $1 \mathrm{~V}$ control signal. The lead compensator increases the system order to two and we select our desired dominant poles with an attenuation of twice that of the first-order pole and a damping ratio of 0.707. Following the root locus method of [5], the lead compensation pole and zero are placed at -6.5 and -2.43 , respectively. The resulting improvement due to the addition of lead compensation on the step response is presented in figure 4.2.

\subsection{Pole Cancellation}

The "pole" cancellation controller is so named because it compensates for thruster dynamics by canceling the apparent lag pole with a corresponding zero. The "pole" cancellation controller avoids the limitations of the lead compensator by adjusting 


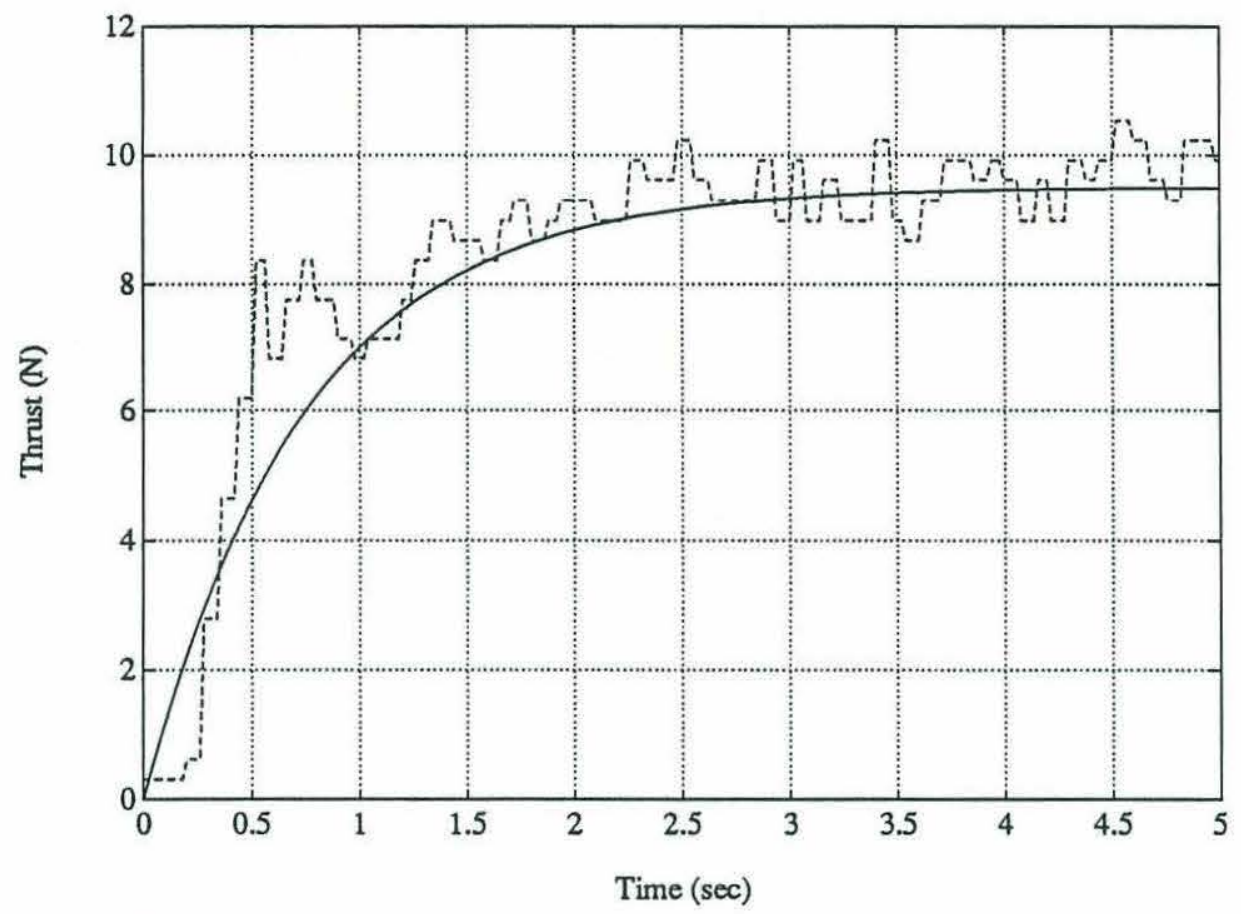

Figure 4.1: Example of $1 \mathrm{~V}$ input step response with a corresponding first-order lag response.

[ - first-order lag response - - actual response]

for the apparent shifts in the first-order lag characteristics. The structure of a lead compensator is driven by the requirement that it be implemented with a passive analog circuit, however, the actual desired effect is the cancellation of an unwanted pole with a corresponding zero. In a digital implementation, pole cancellation can be accomplished by adding a zero but without adding another "fast" pole as is the case for lead compensation. Additionally, the digital implementation is not constrained to a single, representative first-order time constant.

"Pole" Cancellation Block Diagram

$$
\mathrm{u} \rightarrow \mathrm{k} \rightarrow \mathrm{Ts+1} \rightarrow \mathrm{v}
$$

Corresponding Differential Equation

$$
\mathrm{v}=\mathrm{k}(\mathrm{u}+\mathrm{T} \dot{\mathrm{u}})=\mathrm{k}\left(\mathrm{u}+\frac{\dot{\mathrm{u}}}{\lambda_{\mathrm{v}}}\right)
$$




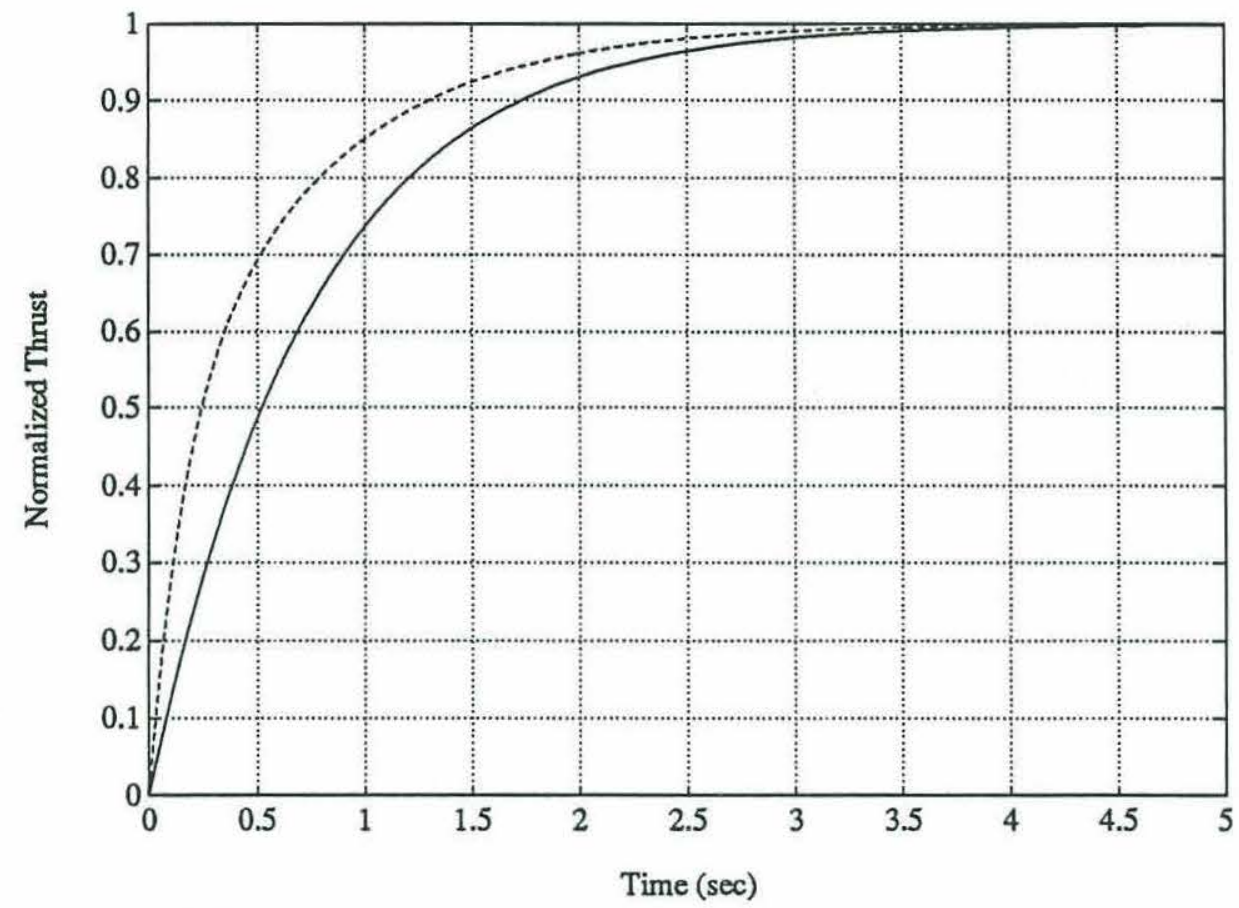

Figure 4.2: Improvement in the thruster step response with lead compensation.

[- uncompensated -- compensated]

where,

$\mathrm{u}$ is the desired control input (thrust).

$\mathrm{v}$ is the compensated control signal $\left(\mathrm{V}_{\mathrm{C}}\right)$.

$\mathrm{T}$ is the effective time constant.

$\lambda_{\mathrm{v}}$ is the inverse of the time constant.

$\mathrm{k}$ is the static gain (thrust-to-torque).

The pole cancellation method requires the evaluation of the time derivative of the commanded control signal, ù. ù can be determined explicitly if the commanded control signal for the thruster is known a priori as when there is a predefined force trajectory for the associated vehicle, however this is not normally the case. ú may also be determined by differentiating the vehicle control law resulting in some terms which are 
known explicitly and some may be determined by numerical differentiation. For example, if the vehicle control law is,

$$
\begin{array}{r}
\mathrm{u}=-\mathrm{K}_{\mathrm{p}} \tilde{\mathrm{x}}-\mathrm{K}_{\mathrm{d}} \dot{\tilde{\mathrm{x}}} \\
\dot{\mathrm{u}}=-\mathrm{K}_{\mathrm{p}} \dot{\tilde{\mathrm{x}}}-\mathrm{K}_{\mathrm{d}} \ddot{\tilde{x}}
\end{array}
$$

where,

$$
\tilde{\mathrm{x}}=\mathrm{x}-\mathrm{x}_{\mathrm{d}}(\text { actual }- \text { desired state })
$$

$\dot{\tilde{\mathbf{x}}}$ is known from the state measurement and $\ddot{\tilde{\mathrm{x}}}$ may be determined by numerical differentiation with a known error bound taking the form of a disturbance.

$\lambda_{\mathrm{v}}$ represents the location of the apparent first-order thruster "pole" and is considered a function of the magnitude of the commanded input. $\lambda_{v}\left(\mathrm{~V}_{C}\right)$ was determined empirically by matching the step response of a first-order system to the observed thruster step response, an example of which is shown in figure 4.1. $\lambda_{\mathrm{v}}\left(\mathrm{V}_{\mathrm{C}}\right)$ was determined for the full range of thruster operation and matched to the functional form,

$$
\lambda_{\mathrm{v}}\left(\mathrm{V}_{\mathrm{C}}\right)=0.235 \sqrt{\mathrm{V}_{\mathrm{C}}}
$$

which matches the empirical data well as indicated in figure 4.3.

Combining this functional form for $\lambda_{\mathrm{v}}\left(\mathrm{V}_{\mathrm{C}}\right)$ with the "pole" cancellation differential equation yields the desired control law,

$$
\mathrm{V}_{\mathrm{C}}=\mathrm{k}\left(\mathrm{u}+\frac{\dot{\mathrm{u}}}{0.235 \sqrt{\mathrm{V}_{\mathrm{C}}}}\right)
$$

$$
\mathrm{k}=\frac{\alpha}{\beta C_{\mathrm{t}}}=0.045 \text { volts } / \mathrm{N} \text { as determined in the model verification. }
$$

The above control law was derived strictly from empirical data but a comparison with the developed model illustrates the physical basis for its form.

Recall the thruster dynamic state and output equations from chapter 2, 


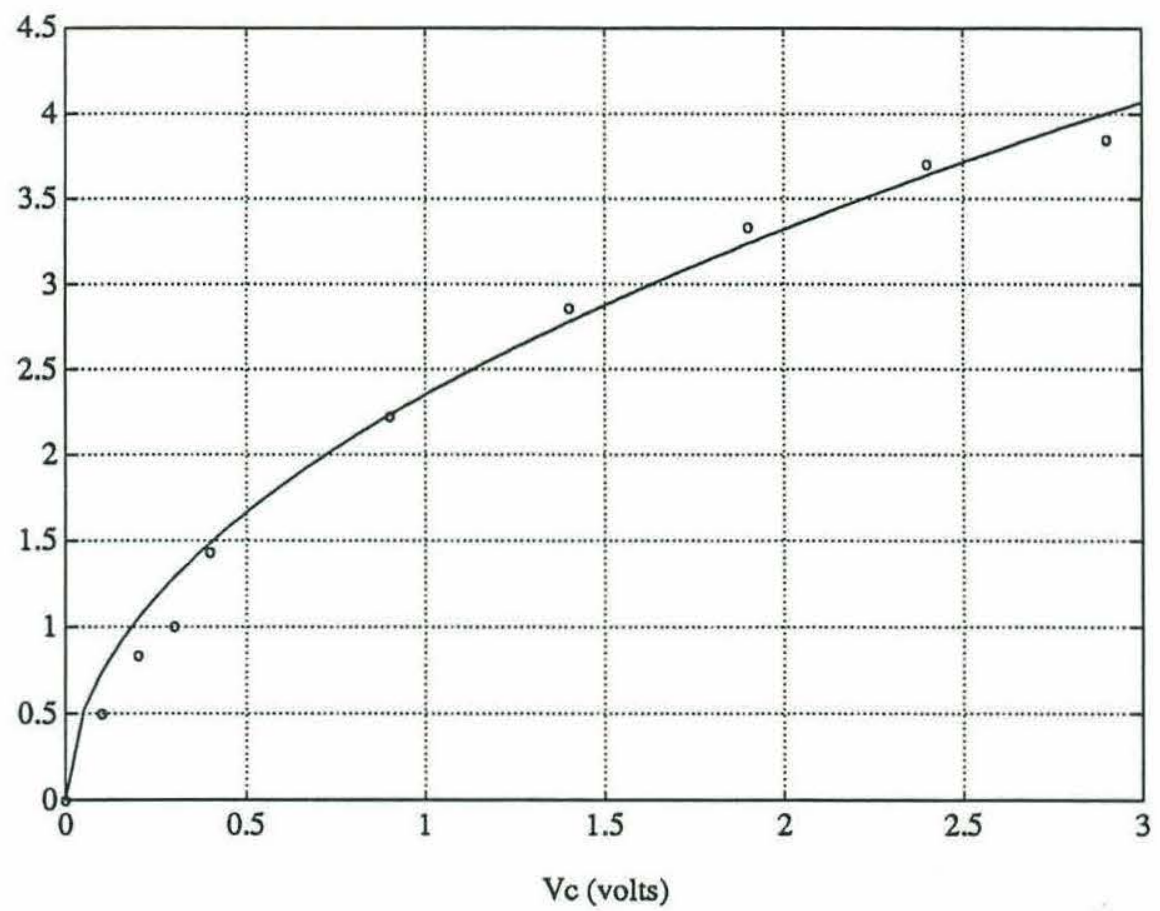

Figure 4.3: Functional form of $\lambda_{v}$ matched to empirical data.

$$
\begin{aligned}
& \dot{\omega}=\beta \mathrm{V}_{\mathrm{C}}-\alpha \omega|\omega| \\
& \text { Thrust, } \mathrm{u}=\mathrm{C}_{\mathrm{\imath}} \omega|\omega|
\end{aligned}
$$

These equations can be alternately presented with the thrust, $u$ as the dynamic state variable,

$$
\frac{\dot{\mathrm{u}}}{2 \sqrt{\mathrm{C}_{\mathrm{t}}}} \sqrt{\mathrm{u}}=\beta \mathrm{V}_{\mathrm{C}}-\frac{\alpha}{\mathrm{C}_{\mathrm{t}}} \mathrm{u}
$$

substituting the static gain relation from the model verification,

$$
\mathrm{u}=\mathrm{C}_{\mathrm{t}} \frac{\beta}{\alpha} \mathrm{v}_{\mathrm{C}}
$$

yields the control law,

$$
V_{C}=\frac{\alpha}{\beta C_{t}}\left\{u+\frac{\dot{u}}{2 \sqrt{\alpha \beta \sqrt{V_{C}}}}\right\}
$$


The derivation of a controller equivalent to the "pole" cancellation controller from the thruster dynamic model reveals the underlying basis for its functional form. Additionally, it provides a parametric form for the apparent first-order lag coefficient, $\lambda_{v}$.

$$
\lambda_{\mathrm{v}} \approx 2 \sqrt{\alpha \beta} \sqrt{\mathrm{V}_{\mathrm{C}}}
$$

This expression can be used as a basis for selecting thruster characteristics which minimize the apparent first-order dynamics.

\subsection{Sliding Controller}

The simple controller designs presented thus far did not utilize the thruster model development. To directly take advantage of the physical nature of the thruster dynamics, a model-based controller is required. Given the perfect measurement of the thruster dynamic state and a perfect model, the input torque can be directly computed and applied, however, this computed torque method may quickly fail in the presence of model uncertainty, measurement noise, computational delays and disturbances. Analysis of the effects of these nonidealities are further complicated by nonlinear dynamics. The issue becomes one of ensuring a nonlinear dynamic system remains robust to nonidealities while minimizing tracking error.

One method able to deal directly with system nonlinearities is referred to as Variable Structure Systems (VSS) and has received considerable attention in Soviet literature. Within VSS theory, sliding modes arise in systems with discontinuous control action with the conditions for their existence specified by Lyapunov stability criteria. Sliding mode theory has been extended to systems with continuous control action and has been further extended to included adaptation to system parametric uncertainty [6]. Application of adaptive sliding mode theory permits precise tracking performance while 
being robust to modeling errors and disturbances. The controller design presented follows closely the development of sliding controllers in [6] and [7].

Sliding Modes are defined as a special kind of motion in the phase space of a dynamic system along a hyperplane for which the control action has discontinuities. This special motion will exist if the state trajectories in the vicinity of the control discontinuity are directed toward the hyperplane [8]. If a sliding mode is properly introduced into a system's dynamics through active control, system behavior will be governed by the selected dynamics on the hyperplane, despite disturbances, nonlinearities, time-variant behavior and modeling uncertainties [9].

With propeller angular velocity, $\omega$ as our thruster state measurement, the thruster dynamic model can be written as,

$$
\begin{aligned}
& \dot{\omega}=\beta \tau-\alpha \omega|\omega| \\
& \text { - or - } \\
& \mathrm{h} \omega=\tau-\mathrm{c} \omega|\omega|
\end{aligned}
$$

where,

$\tau$ is the input torque.

$\alpha$ and $\beta$ are constants determined by the thruster physical configuration.

A sliding surface, $\mathrm{s}$ (in this case, a point) is selected based on simple linear dynamics,

$$
\mathrm{s}=\widetilde{\omega}=\omega-\omega_{\mathrm{d}}
$$

where,

$\omega_{\mathrm{d}}$ is the desired angular velocity.

Perfect tracking is then achieved by the state trajectory remaining at the sliding point $(s=0)$. 
Having selected the sliding point, the condition (referred to as the sliding condition) necessary to constrain trajectories to be directed toward the sliding point must be determined. The sliding condition is satisfied if a quadratic form of $\mathrm{s}$ can be established as a Lyapunov function of the closed loop system [7].

$$
\begin{gathered}
\mathrm{V}=\frac{1}{2} \mathrm{~h} \mathrm{~s}^{2} \\
- \text { and }- \\
\dot{\mathrm{V}}=\mathrm{sh} \dot{\mathrm{s}} \leq 0
\end{gathered}
$$

Imposing the sliding condition on the dynamic model and sliding point yields the control law,

$$
\begin{gathered}
\mathrm{s}=\widetilde{\omega} \\
\mathrm{h} \dot{\mathrm{s}}=\mathrm{h} \dot{\omega}=\mathrm{h}\left(\dot{\omega}-\hat{\omega}_{\mathrm{d}}\right)
\end{gathered}
$$

substituting the system dynamics,

$$
\mathrm{h} \dot{\mathrm{s}}=\tau-\mathrm{c} \omega|\omega|-\mathrm{h} \dot{\omega}_{\mathrm{d}}
$$

the best approximation of a continuous control law that would achieve $h \dot{s}=0$ is,

$$
\hat{\imath}=\hat{c} \omega|\omega|+\hat{h} \dot{\omega}_{d}
$$

where,

^indicates an estimate.

To satisfy the sliding condition despite model uncertainty, a discontinuous control law is required. This can be achieved by adding a term to the approximate continuous control law which is discontinuous across the sliding point.

$$
\tau=\hat{c} \omega|\omega|+\hat{h}\left\{\omega_{\mathrm{d}}-\mathrm{K} \operatorname{sign}(\mathrm{s})\right\}
$$

where,

$$
\operatorname{sign}(s) \equiv\left\{\begin{array}{r}
1 \mathrm{~s}>0 \\
-1 \mathrm{~s}<0
\end{array}\right.
$$


$\mathrm{K}$ is selected large enough to offset the model dynamic uncertainty, ensuring the sliding condition remains satisfied.

Though the discontinuous control law provides for perfect tracking despite nonidealities, it exhibits undesirable chattering around the sliding point. Chattering can be avoided by smoothing the control law within a thin boundary of the sliding point [7].

This idea of a boundary layer and continuous control law seem to violate one of the basic requirements for the existence of a sliding mode; discontinuous control action across the sliding surface, however the boundary layer concept is consistent with the existence of a sliding mode if one examines the source of the chattering it is formulated to eliminate.

Rapid switching in the vicinity of a sliding surface results because the ideal model is only an approximation of the actual plant, neglecting time constants of servomechanisms, sensors and data processors as well as other high order plant dynamics [8]. The frequency of chattering is governed by the high frequency nature of these uncontrolled dynamic nonidealities. By establishing a boundary layer around the sliding surface, we are in effect introducing a dynamic nonideality over which we have control. Hence, the frequency of oscillations in the vicinity of the boundary layer can be made low enough to avoid excitation of high order plant dynamics. The continued existence of a sliding mode in the presence of this controlled dynamic nonideality can be verified as in [8] for uncontrolled dynamic nonidealities. Briefly, if the system with the boundary layer retains the form of ideal sliding as the boundary layer thickness tends to zero, sliding mode behavior is verified.

The dynamic nonideality introduced is in the form of a first-order filter in $\mathrm{s}$ when inside the boundary layer. This can be accomplished by replacing the discontinuous control law term by the continuous term, $\mathrm{K}$ sat $(\mathrm{s} / \phi)$ where,

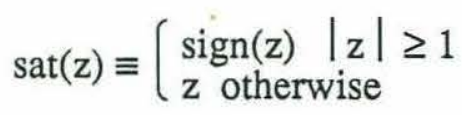


Inside the boundary layer, the dynamics of s should take the form,

$$
\dot{\mathrm{s}}+\lambda \mathrm{s}=\mathrm{bu}
$$

where,
$\mathrm{u}$ is the input.
$\mathrm{b}$ is the input gain.
$\lambda$ is the bandwidth.

which is verified by substituting our modified control law inside the boundary layer into the dynamic equation for the sliding point,

$$
\begin{gathered}
\mathrm{h} \dot{\mathrm{s}}=\tau-\mathrm{c} \omega|\omega|-\mathrm{h} \dot{\omega}_{\mathrm{d}} \\
\mathrm{h} \dot{\mathrm{s}}=\hat{\mathrm{c}} \omega|\omega|+\hat{h} \dot{\omega}_{\mathrm{d}}-\hat{\mathrm{h}} \mathrm{K} \frac{\mathrm{s}}{\phi}-\mathrm{c} \omega|\omega|-\mathrm{h} \dot{\omega}_{\mathrm{d}} \\
\mathrm{h} \dot{\mathrm{s}}=-\hat{\mathrm{h}} \mathrm{K} \frac{\mathrm{s}}{\phi}+\mathrm{Y} \tilde{\mathrm{a}}
\end{gathered}
$$

where,

$$
\begin{aligned}
& \mathbf{a}=[\mathrm{c} \mathrm{h} \\
& ]^{\mathrm{T}} \\
& \tilde{\mathbf{a}} \equiv \hat{\mathbf{a}}-\mathbf{a} \text { is the parametric uncertainty } \\
& \mathbf{Y}=\left[\begin{array}{ll}
\omega|\omega| \omega_{\mathrm{d}}
\end{array}\right] \\
& \qquad \dot{\mathrm{s}}+\frac{\mathrm{K}}{\phi} \mathrm{s}=\hat{\mathrm{h}}^{-1} \mathbf{Y} \tilde{\mathbf{a}}
\end{aligned}
$$

This implies that inside the boundary layer the bandwidth is established by $\frac{K}{\phi}$ with s dynamics driven by the dynamic uncertainty, $\mathbf{Y}$ ã.

The sliding controller with a boundary layer yields the control law,

$$
\tau=\hat{c} \omega|\omega|+\hat{h} \omega_{d}-\hat{h} K \operatorname{sat}(s / \phi)
$$




\subsubsection{Adaptive Sliding Controller}

The sliding controller design can be made adaptive to parametric uncertainty by coupling it to on-line parameter estimation which is based on the algebraic distance of the current state to the boundary layer. [6]. This structure leads naturally to active adaptation only when the system is outside the boundary layer, avoiding the long term drift frequently experienced in parameter estimation schemes [6]. The developed adaptive controller structure is based on the premise that there be no adaptation to that which can be modeled but adapt only to the complex dynamic effects which cannot be simply modeled.

Inside the boundary layer, the s dynamics are,

$$
\dot{\mathrm{s}}+\frac{\mathrm{K}}{\phi} \mathrm{s}=\hat{\mathrm{h}}^{-1} \mathbf{Y} \tilde{\mathbf{a}}
$$

in steady state,

$$
\begin{gathered}
\mathrm{s}=\hat{\mathrm{h}}^{-1} \frac{\phi}{\mathrm{K}} \mathbf{Y} \mathbf{a} \\
\text { - but }-
\end{gathered}
$$

$\mathrm{K}$ was selected to be greater than the dynamic uncertainty $\hat{h}^{-1} \mathbf{Y}$ ã, hence,

$$
\mathrm{s} \leq \phi
$$

This indicates that once in the boundary layer, $\mathrm{s}$ remains there. $\mathrm{s}$ will be driven out of the boundary layer only when the actual dynamic uncertainty exceeds our current estimate. Therefore, we should only adapt when outside the boundary layer and in proportion to the distance to the boundary layer.

To ensure the stability of the adaptive closed loop system, the parameter estimation scheme must preserve the attractiveness of the boundary layer. With this in mind, a Lyapunov function candidate is selected of the form, 


$$
\mathrm{V}=\frac{1}{2}\left[\mathrm{~s}_{\Delta} \mathrm{h} \mathrm{s}_{\Delta}+\tilde{\mathbf{a}}^{\mathrm{T}} \Gamma \tilde{\mathbf{a}}\right]
$$

and sliding condition,

$$
\dot{V}=\left[s_{\Delta} h \dot{s}_{\Delta}+\hat{a}^{T} \Gamma \tilde{a}\right] \leq 0
$$

where,

$$
\begin{aligned}
& \mathrm{s}_{\Delta}=\mathrm{s}-\phi \operatorname{sat}(\mathrm{s} / \phi) \\
& \mathrm{s}_{\Delta}=\left(\begin{array}{l}
0 \text { inside the boundary layer } \\
\mathrm{s}-\phi \operatorname{sign}(\mathrm{s}) \text { outside the boundary layer }
\end{array}\right. \\
& \dot{\mathrm{s}}_{\Delta}=\left(\begin{array}{l}
0 \text { inside the boundary layer } \\
\dot{\mathrm{s}} \text { outside the boundary layer }
\end{array}\right. \\
& \dot{\mathbf{a}}=\mathbf{a} \text { since } \mathbf{a} \equiv 0
\end{aligned}
$$

$\Gamma$ is a symmetric, positive definite matrix.

Imposing the sliding condition outside the boundary layer yields the adaptation recursion relation.

$$
\begin{aligned}
& \dot{V}=\left[s_{\Delta}\{\mathbf{Y} \mathbf{a}-\hat{h} K \operatorname{sign}(s)\}+\hat{a}^{\mathrm{T}} \Gamma \tilde{a}\right] \\
& \dot{V}=\left[\left\{s_{\Delta} \mathbf{Y}+\hat{a}^{\mathrm{T}} \Gamma\right\} \tilde{a}-s_{\Delta} \hat{h} K \operatorname{sign}(\mathrm{s})\right]
\end{aligned}
$$

To satisfy the sliding condition,

$$
\begin{gathered}
\mathrm{s}_{\Delta} \mathbf{Y}+\hat{a}^{\mathrm{T}} \Gamma=0 \\
- \text { and }- \\
\hat{\mathbf{a}}=-\mathrm{s}_{\Delta} \Gamma^{-1} \mathbf{Y}^{\mathrm{T}}
\end{gathered}
$$




\subsection{Summary}

The lead compensator designed compensates for the thruster dynamics by adding phase lead directly to the input control signal to be delivered to the thruster motor. Though lead compensation is simple to implement, it is designed around a single operating point and will be unable to compensate for the full range of thruster dynamics.

The "pole" cancellation controller avoids the limitations of the lead compensator by effectively adjusting its operating point. Though it was designed from empirical data obtained through observations of the thruster step response, the "pole" cancellation controller structure is shown to be model-based. The "pole" cancellation controller has the control law,

$$
\mathrm{V}_{\mathrm{C}}=0.045\left(\mathrm{u}+\frac{\dot{\mathrm{u}}}{0.235 \sqrt{\mathrm{V}_{\mathrm{C}}}}\right)
$$

where,

$\mathrm{u}$ and $\mathrm{u}$ are the desired thrust and thrust rate (in $\mathrm{N}$ and $\mathrm{Ns}^{-1}$ ).

$\mathrm{V}_{\mathrm{C}}$ is the command input to the thruster motor (in volts).

The adaptive sliding controller incorporates the thruster dynamic model into a control structure which is robust to model uncertainty within an established bound (inside the boundary layer) and adaptive to uncertainty outside the bound (outside the boundary layer). The sliding controller has the control law,

$$
\mathrm{V}_{\mathrm{C}}=\hat{\mathrm{c}} \omega|\omega|+\hat{\mathrm{h}}\left\{\dot{\omega}_{\mathrm{d}}-\mathrm{Ksat}(\tilde{\omega} / \phi)\right\}
$$

where,

$\hat{\mathrm{c}}$ is the estimate of the thruster model parameter, $\frac{\alpha}{\beta}$.

$\hat{h}$ is the estimate of the thruster model parameter, $\frac{1}{\beta}$.

$\omega_{\mathrm{d}}$ is the desired propeller angular acceleration (in $\mathrm{rad} / \mathrm{s}^{2}$ ). 
Ф is the propeller angular velocity tracking error (in rad/s).

$\omega_{\mathrm{d}}$ and $\omega_{\mathrm{d}}$ are representative of the desired thrust and thrust rate through the relation; Thrust, $\mathrm{u}=\mathrm{C}_{\mathrm{t}} \omega|\omega|$.

$\mathrm{K}$ is a gain established by the model dynamic uncertainty.

$\phi$ is the width of the boundary layer.

$\mathrm{V}_{\mathrm{C}}$ is the command input to the thruster motor (in volts).

When the tracking error, $\widetilde{\omega}$ exceeds the boundary layer, the controller parameters, $\hat{\mathrm{c}}$ and $\hat{\mathrm{h}}$ are updated according to the recursion relations,

$$
\begin{gathered}
\hat{\mathrm{c}}=-\frac{1}{\gamma_{1}} \omega|\omega| \tilde{\omega} \\
\dot{\mathrm{h}}=-\frac{1}{\gamma_{2}} \omega_{\mathrm{d}} \varpi
\end{gathered}
$$

where,

$\gamma_{\mathrm{i}}$ are gains which establish the relative weighting and sensitivity of the parameter updates. 


\section{Chapter 5}

\section{CONTROLLER EVALUATION}

Each of the controllers of Chapter 4 were designed to compensate for the thruster dynamics directly, in essence to alter the apparent dynamics to be closer to that of a thrust servo. Though it can be demonstrated that each controller modifies the uncompensated thruster time and frequency response, the response improvements do not represent the best criteria for comparing controllers. The goal of the thruster dynamic compensation is to improve the tracking performance of a position-controlled underwater vehicle. Therefore the controllers will be evaluated and compared on the basis of tracking performance when coupled to a vehicle.

\subsection{Hybrid Simulation}

Controller performance could be evaluated extensively and quickly utilizing the thruster model and standard digital simulation techniques. Pure simulation however frequently fails to reveal real implementation issues such as processing time delays, measurement noise and resolution, and model uncertainties, just to name a few. To minimize the limitation inherent in simulation, to strengthen the results and reduce the effort required to incorporate a controller into an actual underwater vehicle, a hybrid simulation was developed. 
The hybrid simulation utilizes an actual thruster fitted with appropriate sensors as the actuator for a simulated one degree-of-freedom position-controlled vehicle. This arrangement permitted extensive evaluation without time-consuming actual underwater vehicle operation while addressing many of the implementation issues.

\subsubsection{Vehicle Model}

Two vehicle models were developed using scaled hydrodynamic parameters equivalent to the Deep Submergence Laboratory remotely operated vehicles (ROV's) JASON and its prototype JASON Jr. (JJ). Though the two models share the same form, they represent inertia dominated (JASON) and drag dominated (JJ) underwater vehicles. The hydrodynamic drag is assumed proportional to the square of the vehicle velocity with added mass effects equal to three times the vehicle dry mass. This yields a vehicle dynamic model of the form,

$$
M \ddot{x}=\text { thrust }-C_{D} \dot{x}|\dot{x}|
$$

where,

$M$ is the vehicle effective mass, including hydrodynamic effects.

$C_{D}$ is the drag coefficient.

The model parameters for each vehicle are listed in the table below.

$\begin{array}{lll} & \text { Vehicle 1 (JASON) } & \text { Vehicle 2(JJ) } \\ \mathrm{C}_{\mathrm{D}}[\mathrm{kg} / \mathrm{m}] & 450 & 67 \\ \mathrm{M}[\mathrm{kg}] & 1300 & 340\end{array}$




\subsubsection{Vehicle Position Controller}

A vehicle position controller structure similar to that used in the closed loop control of JASON and JASON Jr. was used in the vehicle simulation. The controller modifies the system dynamics to be that of a critically damped second order system with nonlinear feedforward drag compensation. The resulting vehicle control law is,

$$
\mathrm{u}=\mathrm{C}_{\mathrm{D}} \dot{\mathrm{x}}|\dot{\mathrm{x}}|-2 \lambda \dot{\tilde{\mathrm{x}}}-\lambda^{2} \tilde{\mathrm{x}}
$$

This control law would yield near perfect tracking when coupled to the developed vehicle model if there were no actuator (thruster) dynamics present. Hence, the selected vehicle position-controller provides a consistent and fair basis for controller comparisons.

The vehicle control law is differentiated to provide the desired thrust rate to the "pole" cancellation and adaptive sliding controllers. Differentiation of the control law instead of the desired thrust allows some terms to be expressed explicitly, reducing the error that would be associated with strictly implicit differentiation of the desired thrust. This yields a desired thrust rate of,

$$
\dot{\mathrm{u}}=2 \mathrm{C}_{\mathrm{D}} \ddot{\mathrm{x}}|\dot{\mathrm{x}}|-2 \lambda \ddot{\tilde{\mathrm{x}}}-\lambda^{2} \dot{\tilde{\mathrm{x}}}
$$

where,

$\ddot{x}$ is determined by implicit differentiation of $\dot{x}$.

\subsubsection{Thruster Controller Interfaces}

The system setup for the hybrid simulation is presented schematically in figure 5.1. All the thruster controllers are provided the desired thrust as input. With no compensation, this signal is adjusted by a constant gain and input to the motor controller 


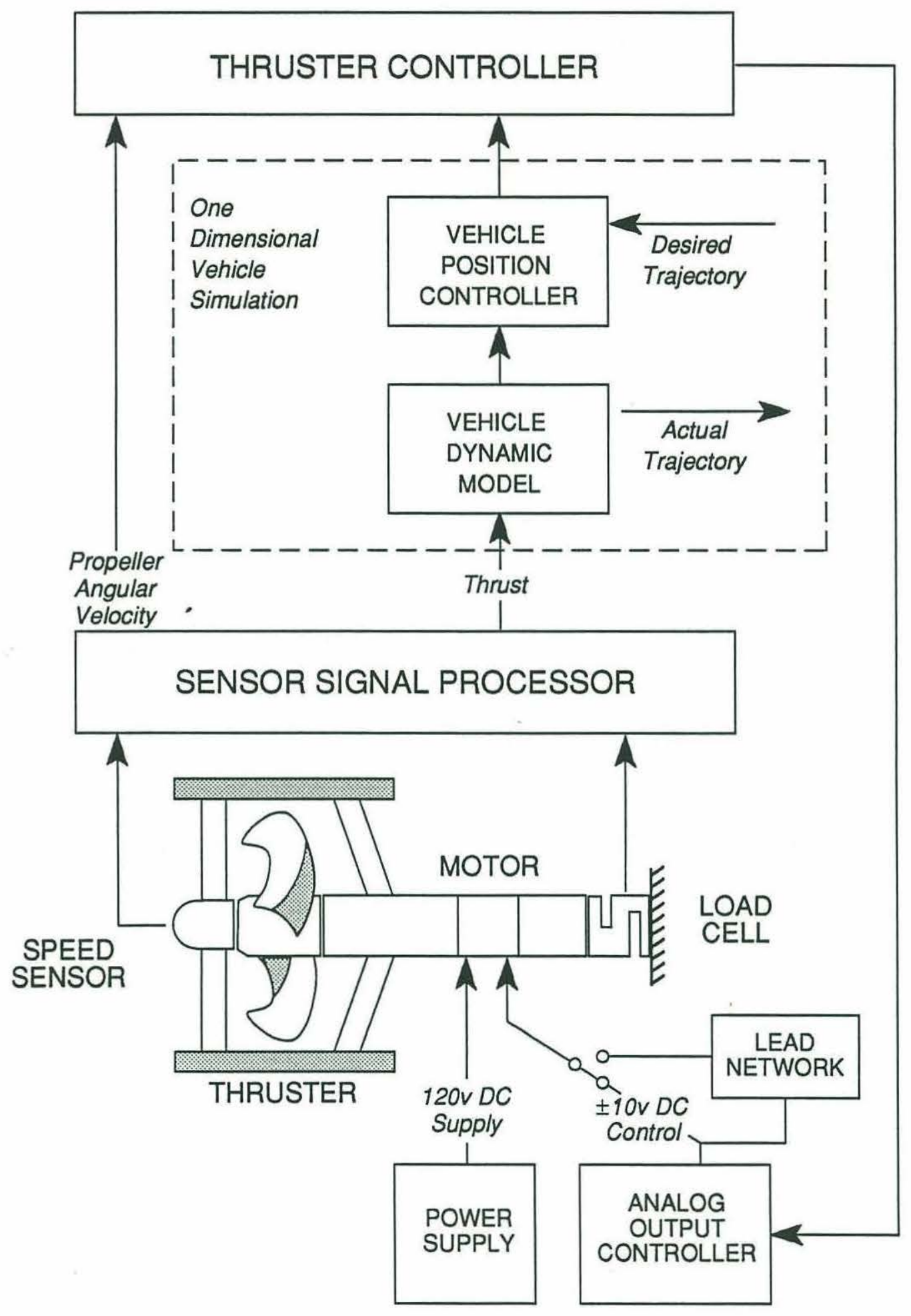

Figure 5.1: System setup for hybrid simulation 
as the desired torque. In the case of the analog lead compensation, the desired thrust input is processed as without compensation but the control voltage to the motor is delivered through the lead network. The lead network consists of a resistor-capacitor (RC) circuit. An amplifier is utilized to correct for the attenuation of the RC circuit and isolate it from the motor controller.

The "pole" cancellation controller is provided the desired thrust and thrust rate from the vehicle controller. A motor control voltage is formed using the control law of chapter 4 and delivered to the thruster.

The adaptive sliding controller receives desired thrust and thrust rate from which the desired propeller angular velocity and acceleration are determined. The actual propeller angular velocity is input from the propeller speed sensor. A motor control voltage is formed using adapted parameter estimates in the control law of chapter 4 and delivered to the thruster.

\subsection{Evaluation Results}

\subsubsection{No Compensation}

The thruster dynamics include two regimes which challenge the performance of each control scheme. The first is motor saturation. The motor controller saturates at a delivered thrust of approximately $125 \mathrm{~N}$ (28 lbf). Though no controller can produce an output which exceeds the capability of the actuator, the controller which provides tracking with minimum control action avoids saturation effects through a greater range of vehicle trajectories. Figure 5.2 demonstrates this effect when no compensation for thruster dynamics is utilized. Though the desired trajectory was within the capability of 


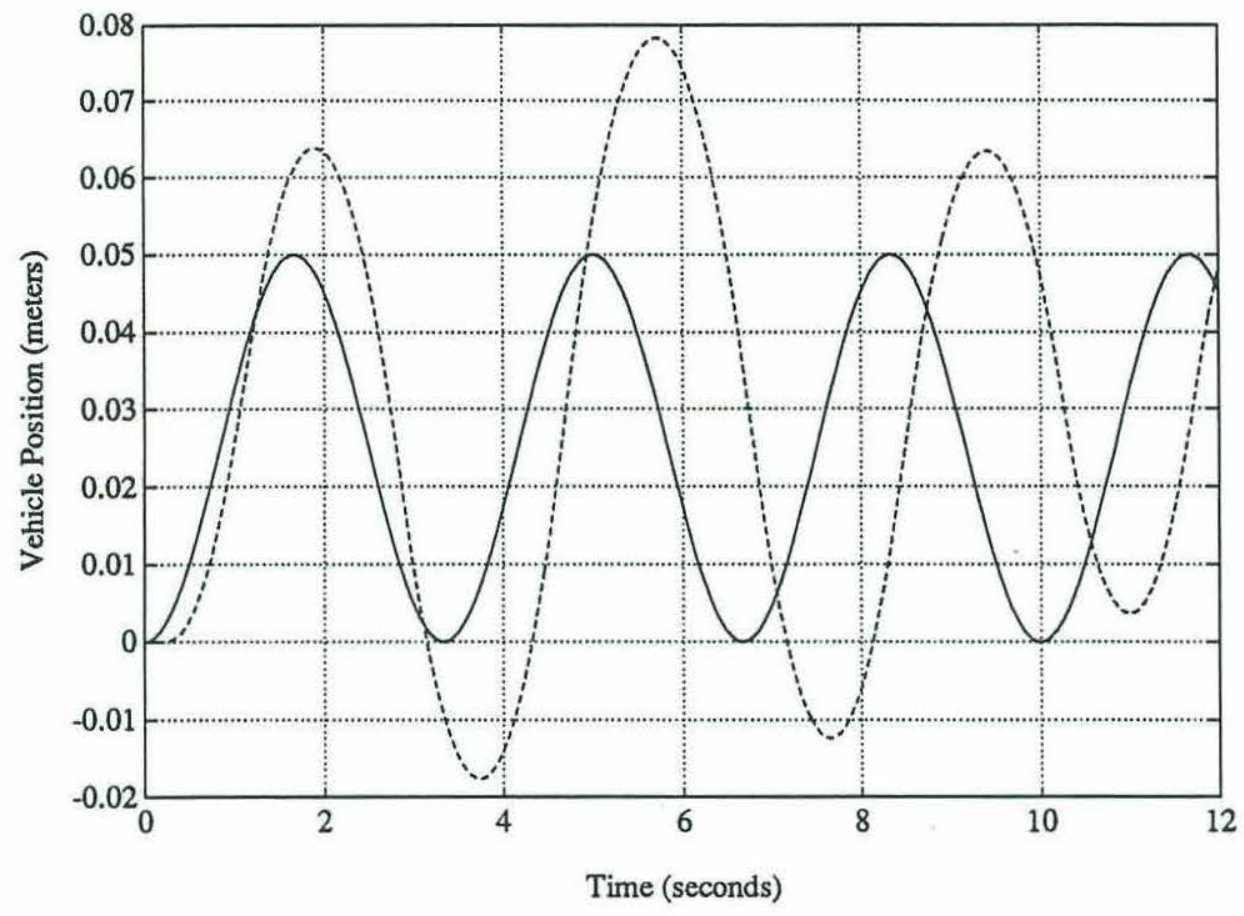

Figure 5.2: Effect of thruster motor saturation without thruster dynamic compensation.

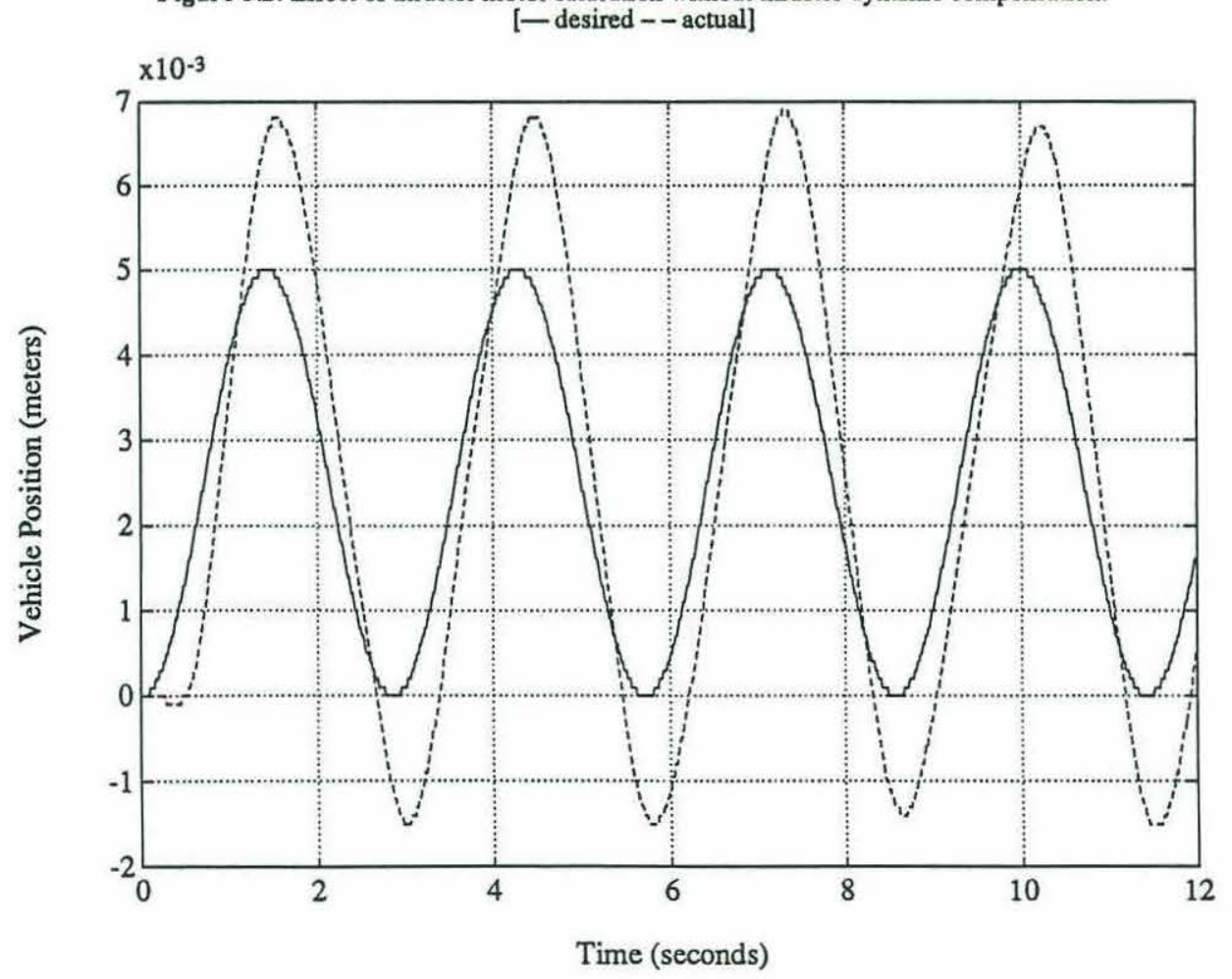

Figure 5.3: Effect of thruster dynamics without thruster dynamic compensation at low thrust. [-desired -- actual] 
the thruster and vehicle, the lack of compensation results in thruster saturation and precluded acceptable tracking performance. Of note, all of the thruster controllers evaluated provided acceptable tracking performance of this same trajectory.

The second regime is at very low thrust where saturation is not a problem but the hydrodynamic "lag" is most prominent. Without compensation for the thruster dynamics, the vehicle tracking is again unacceptable as presented in figure 5.3. Acceptable tracking performance without compensation for the thruster dynamics is achieved only by reducing the bandwidth of the vehicle, effectively reducing the range of trajectories which may be obtained.

\subsubsection{Lead Compensation}

Introducing lead compensation into the motor control significantly improved tracking performance over that obtained without compensation as seen in figure 5.4.

The lead network was designed with the assumption that the thruster dynamics could be characterized by a linear first-order lag represented by the thruster step response to an input control signal of 1 volt. As with any linearization of nonlinear dynamics, performance away from the point of linearization may be degraded. When higher values of thrust are required, the vehicle tracking performance with lead compensation may not be significantly better than without compensation as shown in figure 5.5. This effect was more prominent when the simulated vehicle was inertia dominated as higher thrust was required for similar trajectories.

When very low values of thrust were required, tracking performance with lead compensation was unacceptable as presented in figure 5.6. Note that this is the same desired trajectory as presented in figure 5.3. The reason for this poor performance is again the result of operation away from the point of linearization. The root locus plots of 


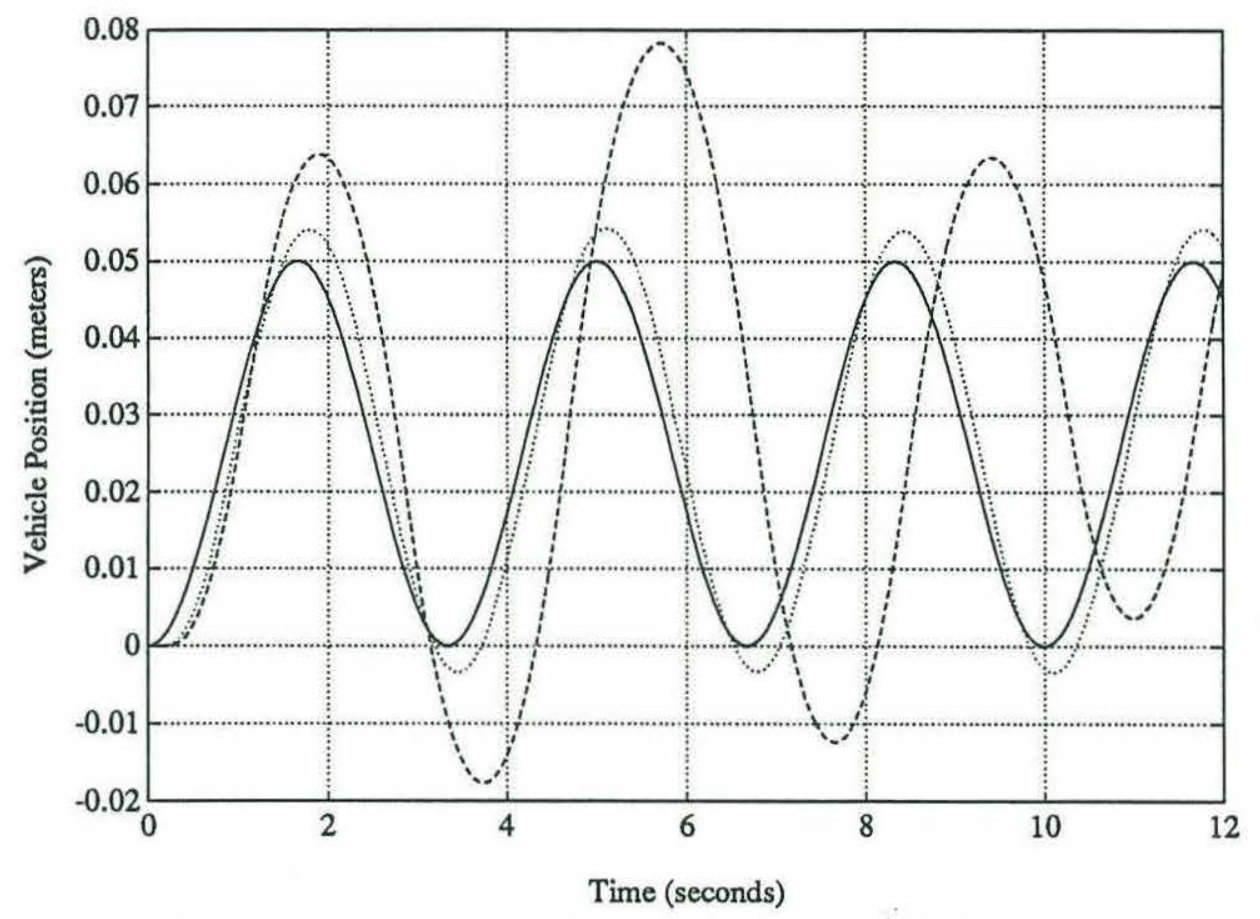

Figure 5.4: Improved vehicle tracking performance obtained with lead compensation. [ - desired -- actual, no compensation $\cdots$ actual, lead compensation]

figure 5.7 demonstrate the consequences of lead compensation designed for a first-order lag which is significantly faster than the actual lag. The root locus confirms the observed phase-lagged, oscillatory behavior of figure 5.6. The low thrust characteristics of the lead compensation presented could be avoided by shifting the design operating point to the maximum observable time constant, however this would significantly reduce its effectiveness in compensating for the thruster dynamics at moderate and higher thrust levels.

\subsection{3 "Pole" Cancellation}

The "pole" cancellation controller is so named because it compensates for thruster dynamics by canceling the apparent lag pole with a corresponding zero. The 

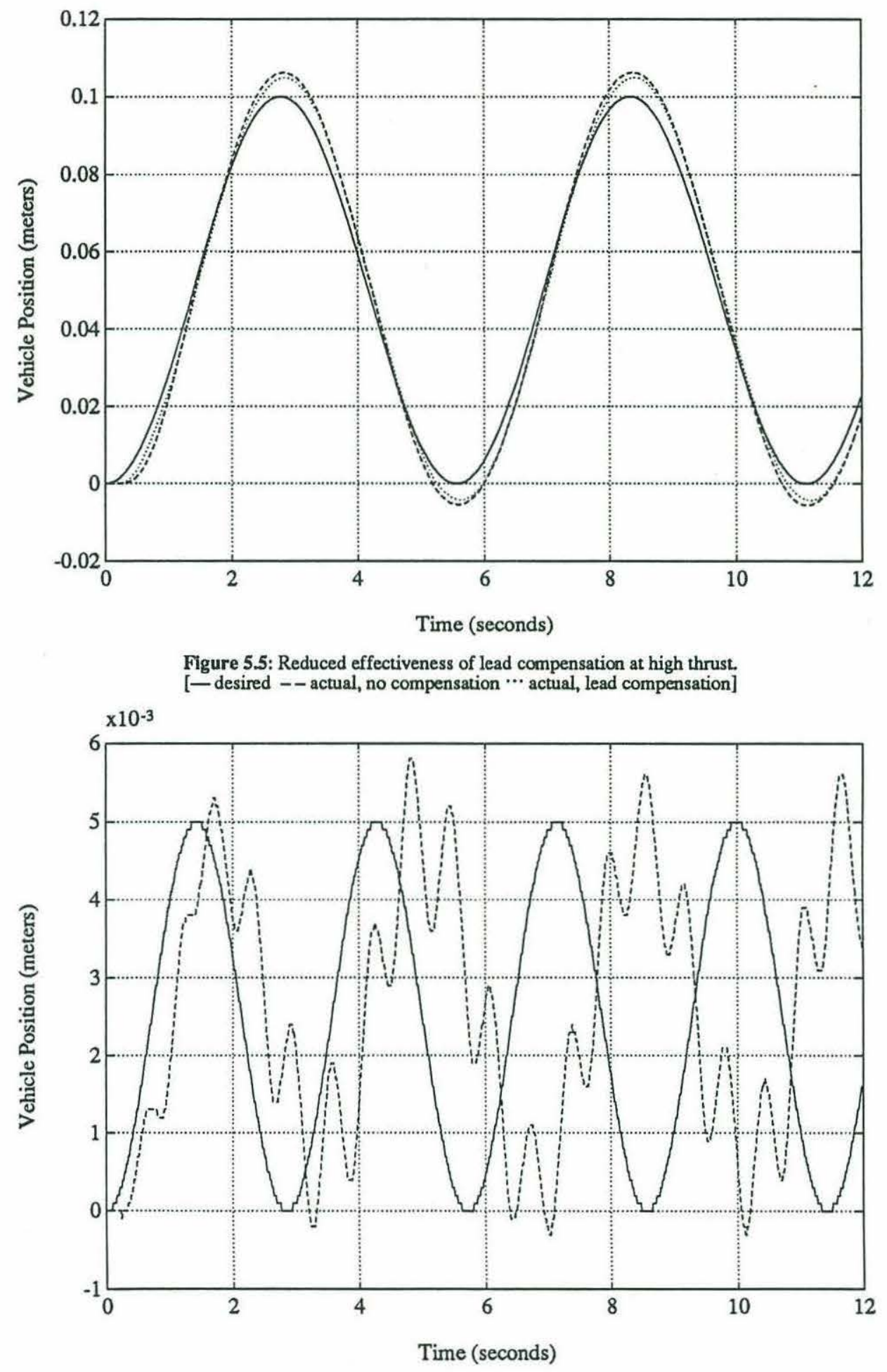

Figure 5.6: Reduced effectiveness of lead compensation at low thrust. [- desired - - actual] 

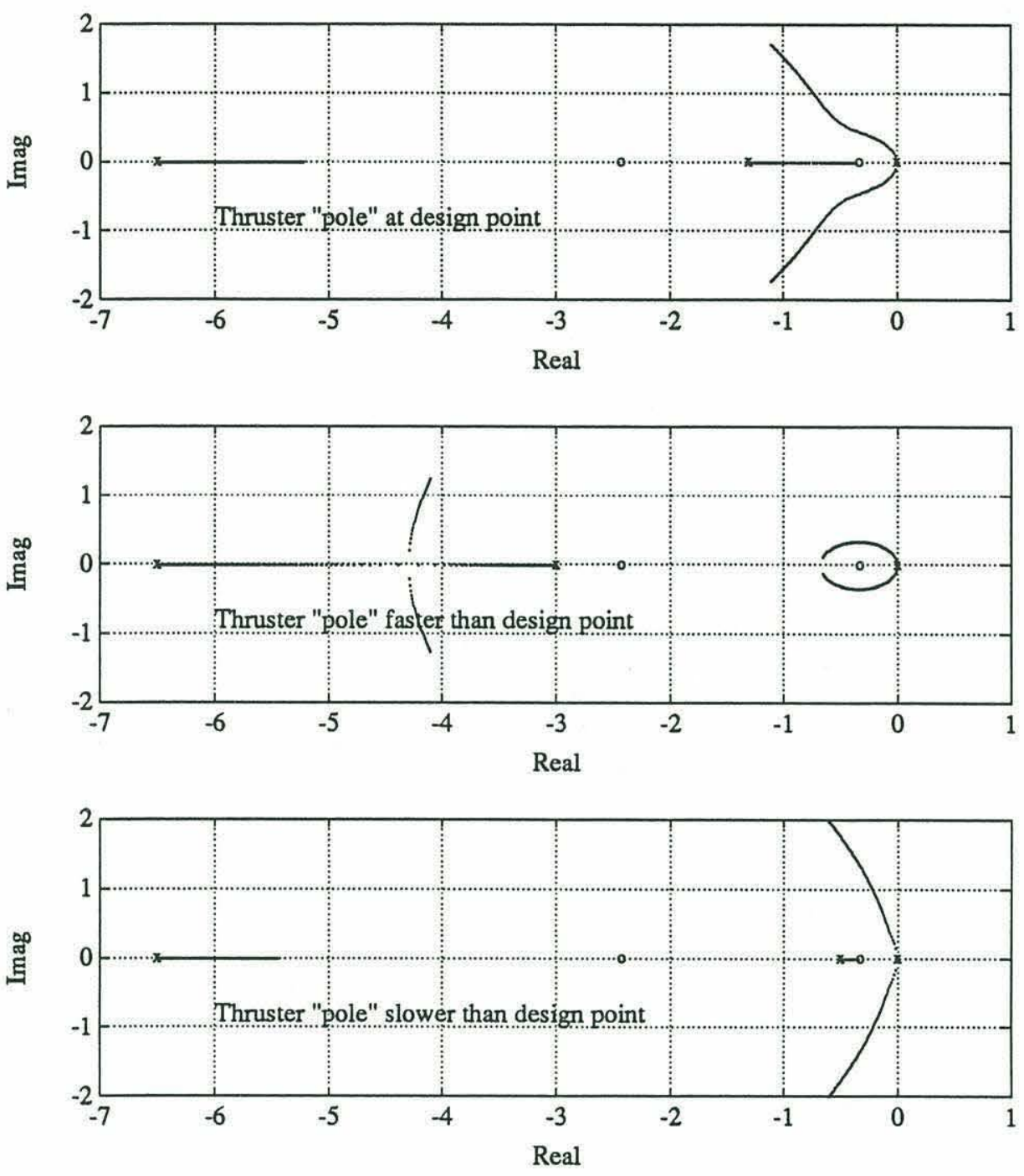

Figure 5.7: Root locus plots demonstrating the results of operating away from the point of lead compensator design and system linearization points.

Plots were obtained assuming the vehicle was an inertia with a velocity controller zero at -0.333 , lead compensator zero at -2.43 and pole at -6.5 , and thruster poles at $-1.3,-3$, and -0.5

"pole" cancellation controller avoids the limitations of the lead compensator by adjusting for the apparent shifts in the first-order lag characteristics. As presented in figure 5.8, the "pole" cancellation controller provides for good tracking performance over the full range of thruster operation. In chapter 4, it was shown that the form of the "pole" cancellation 

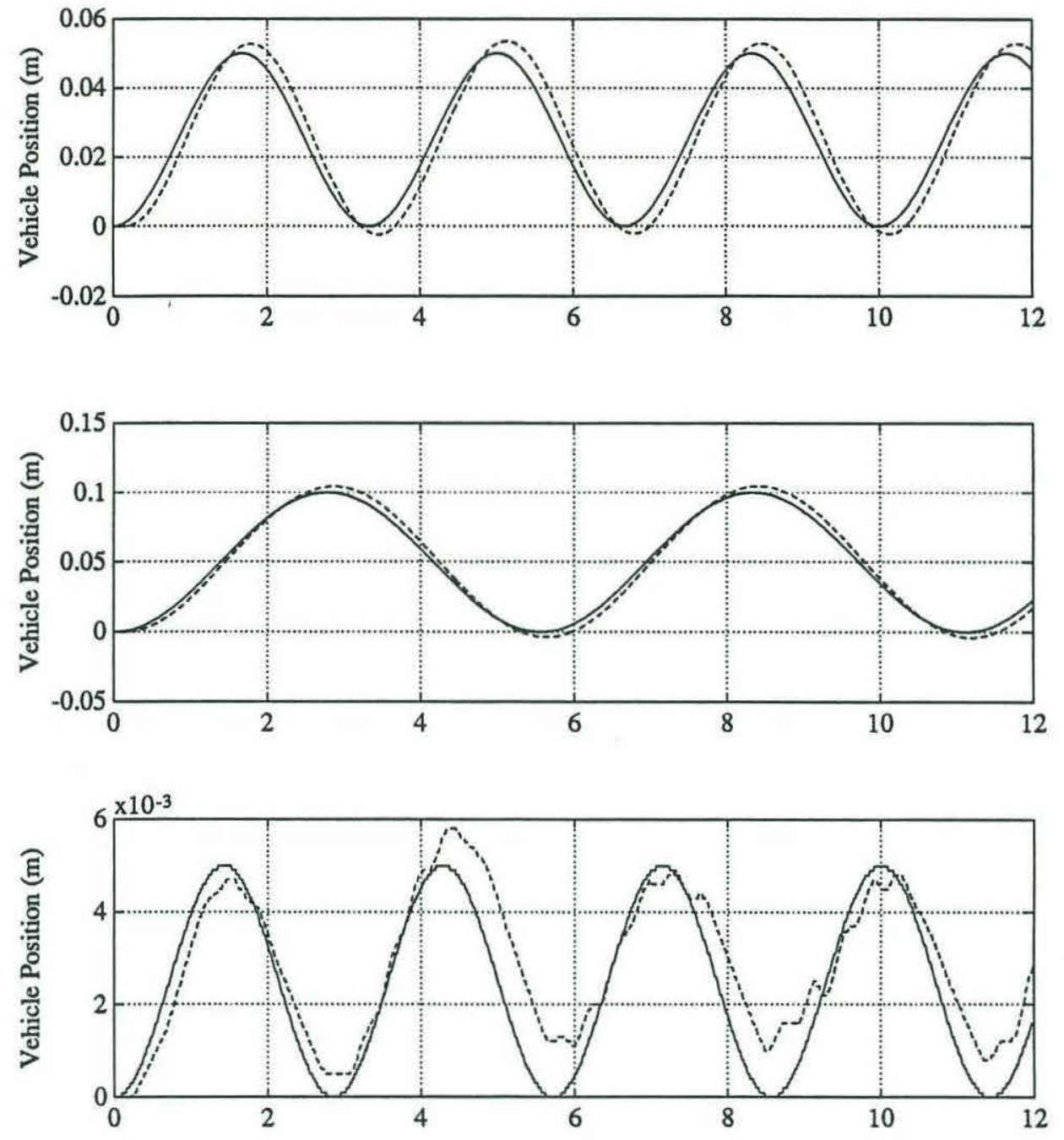

Figure 5.8: Vehicle tracking performance with "pole" cancellation thruster controller. RMS position errors $.0045, .0041$ and .00081 meters, respectively.

controller can be derived from the thruster dynamic model. Hence, the "pole" cancellation controller can be viewed as a model-based computed torque controller. Since the "pole" cancellation controller receives no feedback information of the thruster dynamic state, it is operating in open-loop. The form of the "pole" cancellation controller permits good tracking performance provided the open-loop model remains valid. 


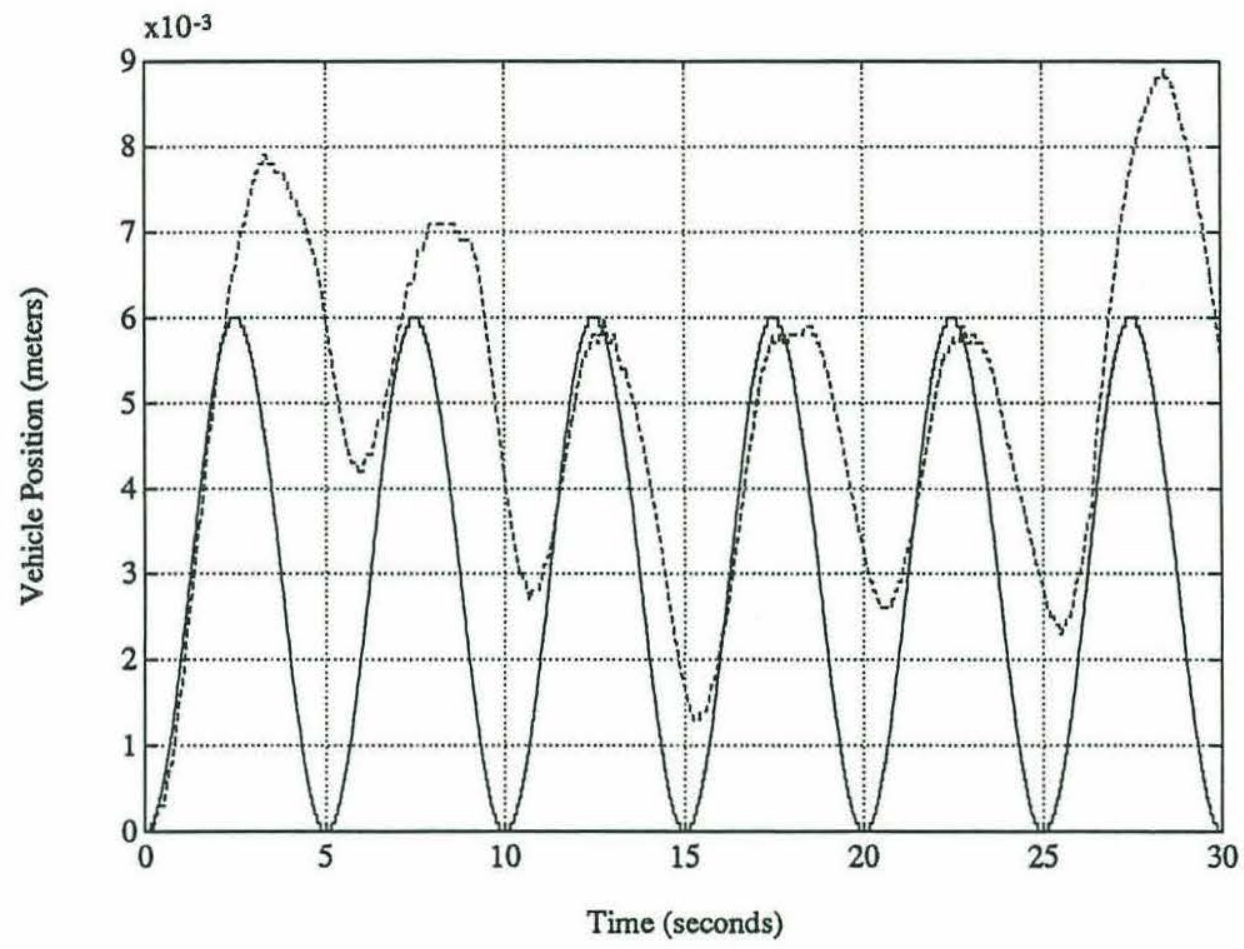

Figure 5.9: Degraded "pole" cancellation thruster control with obstruction in thruster duct outlet. [- desired trajectory - - actual trajectory]
RMS position error is .0028 meters.

The major weakness of the "pole" cancellation approach is its dependence on a precise knowledge of the thruster and hence model parameters. Figure 5.9 demonstrates the effect of a change in the model parameters on tracking performance with "pole" cancellation thruster control. An obstruction was placed in the thruster duct outlet, reducing the effective area. As a result the thruster model basis changed and the tracking performance of the vehicle significantly degraded. Similar degradation in performance could be expected to result from other kinds of more common apparent changes in the model, such as cross-flow and mutual interference. 

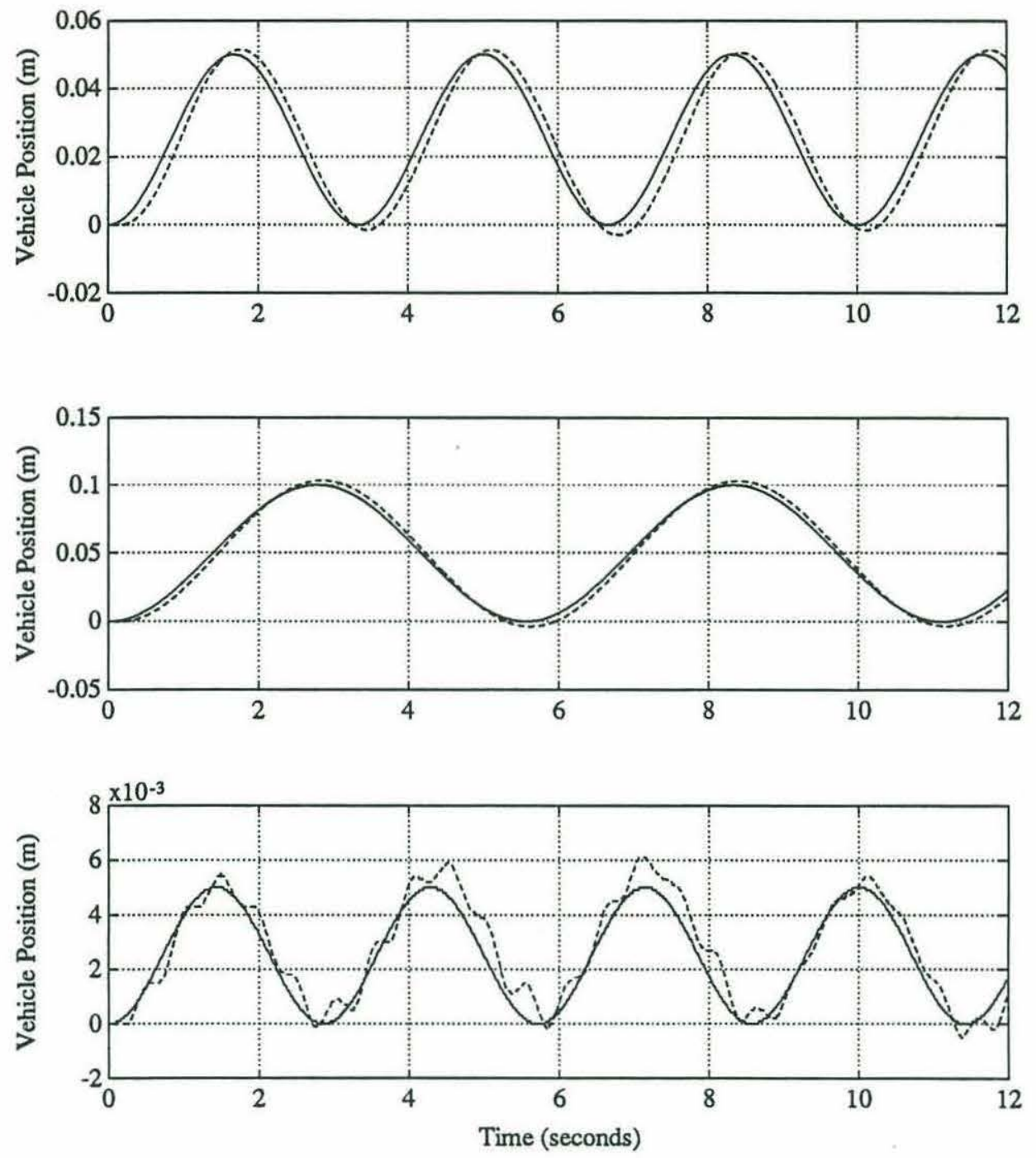

Figure 5.10: Vehicle tracking performance with adaptive sliding thruster controller. [- desired trajectory - - actual trajectory] RMS position errors $.0042, .0036$ and .00059 meters, respectively.

\subsubsection{Adaptive Sliding Controller}

The model-based adaptive sliding controller avoids the limitations of the other controllers evaluated. Good vehicle tracking performance over the full range of thruster 


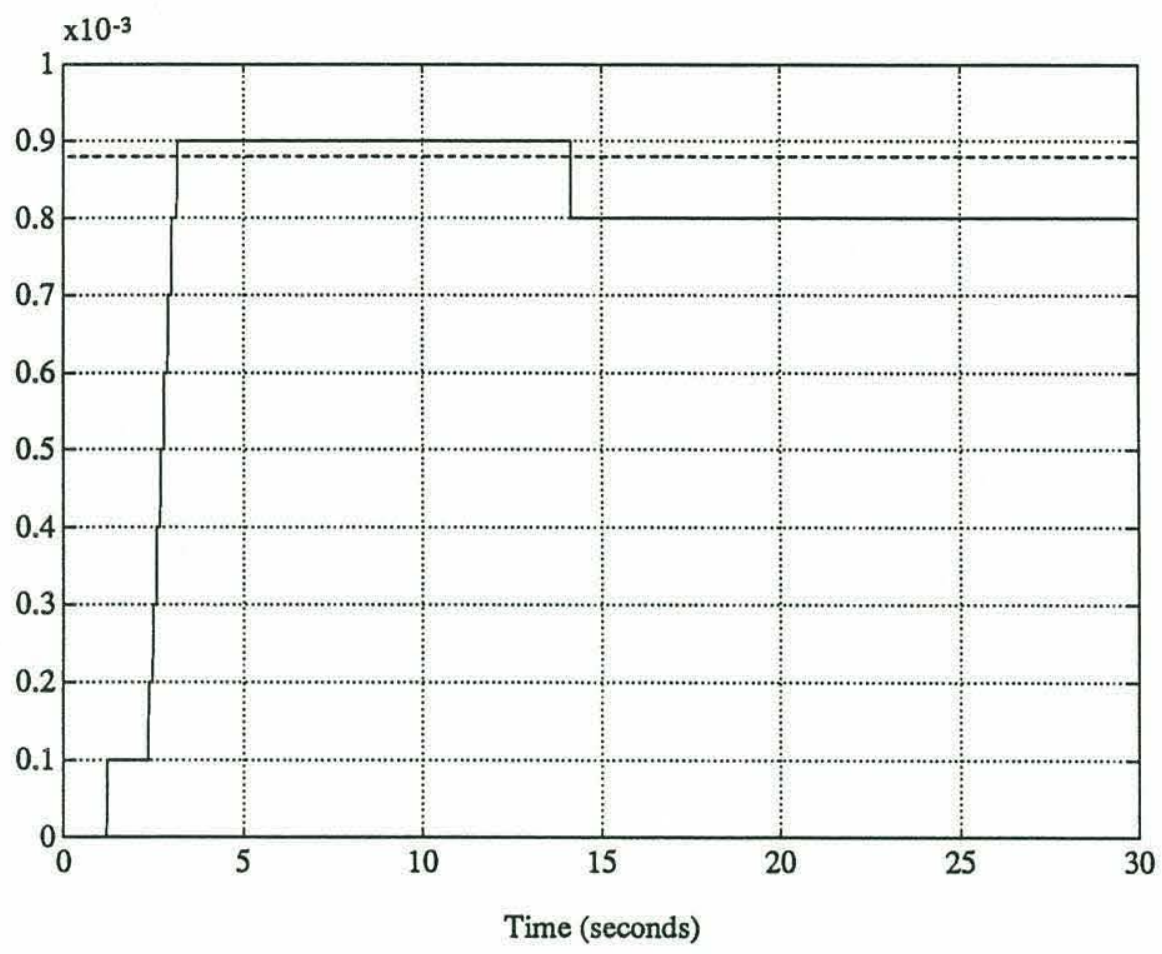

Figure 5.11: Thruster model parameter, c convergence. [- parameter estimate - - actual value]

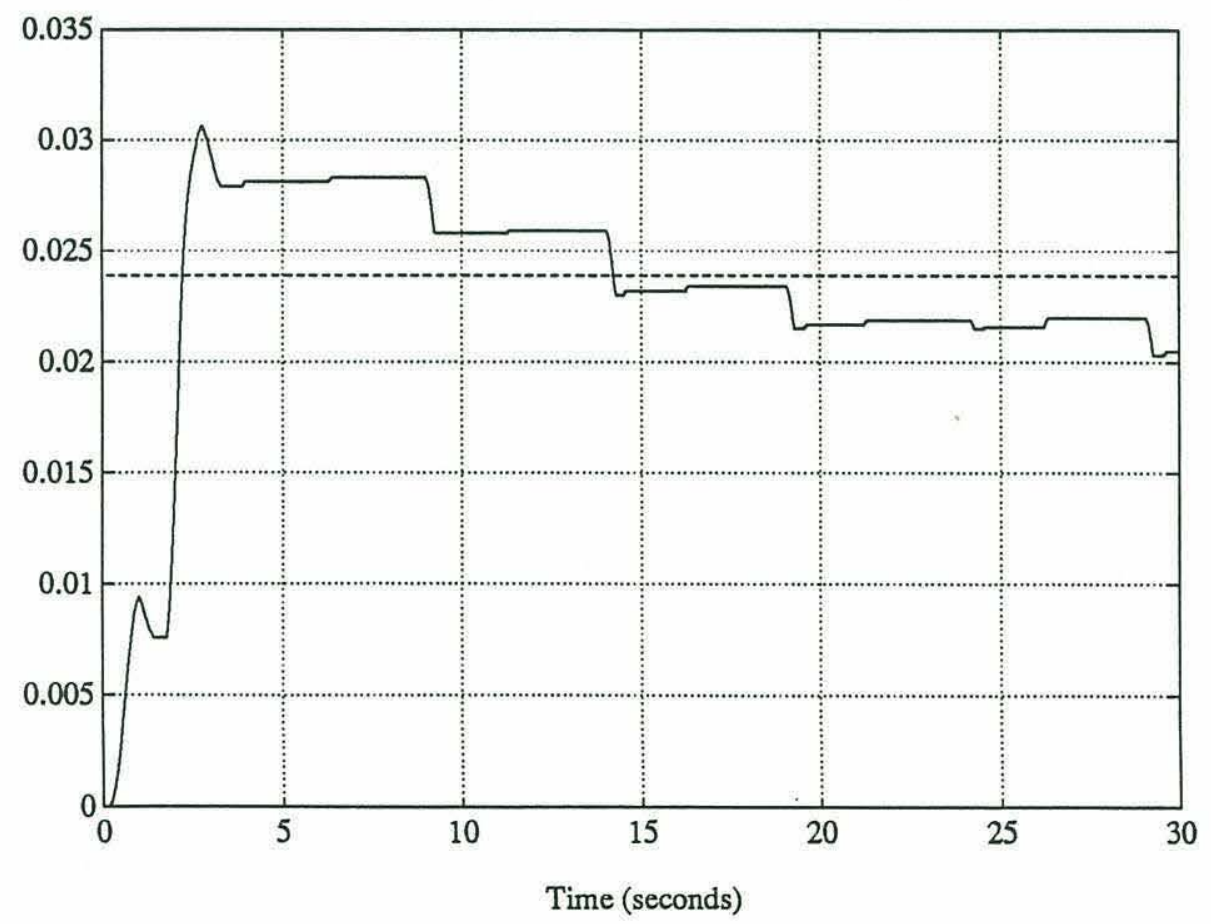

Figure 5.12: Thruster model parameter, $h$ convergence. [- parameter estimate - - actual value] 


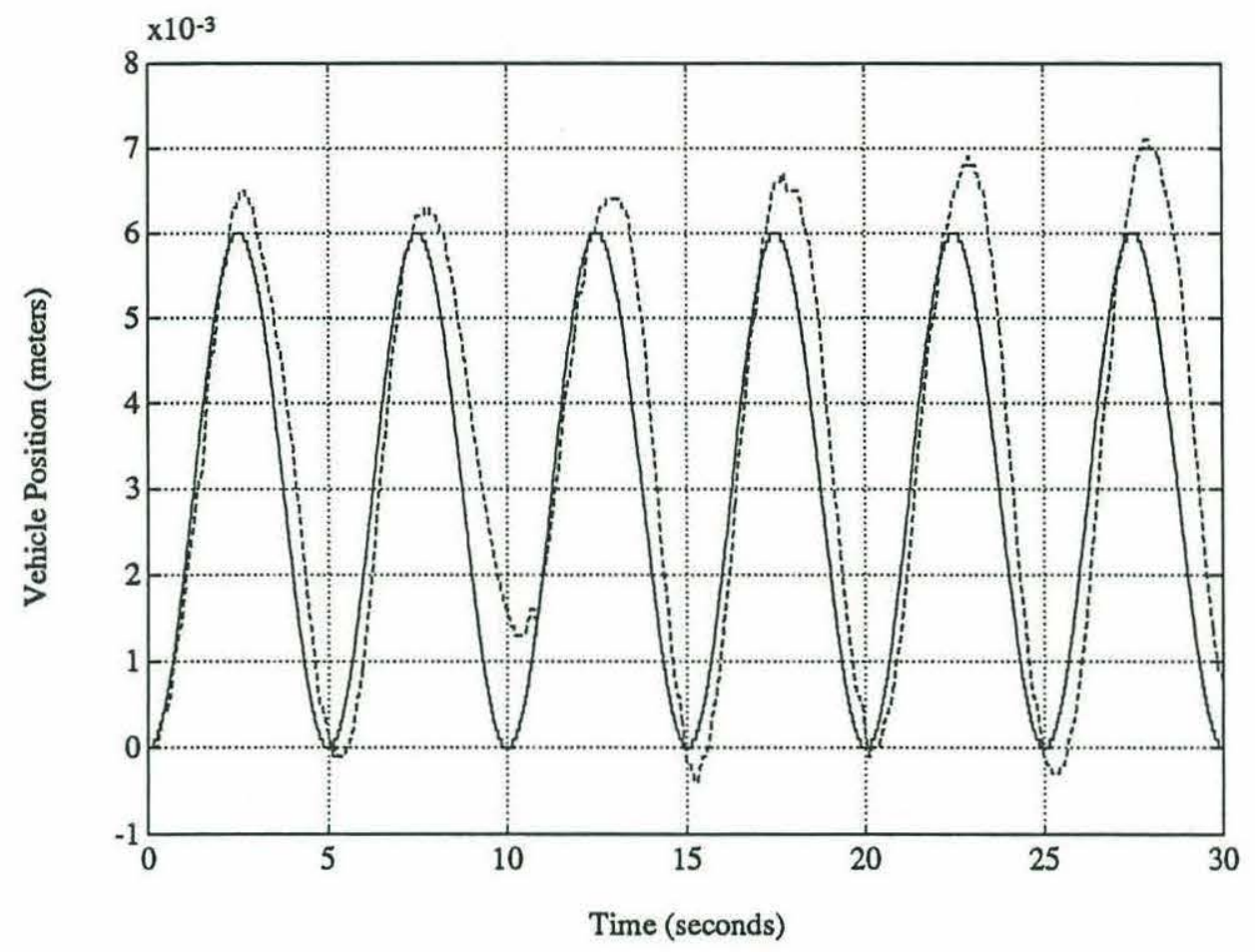

Figure 5.13: Adaptive sliding thruster control with obstruction in the thruster duct outlet. [- parameter estimate -- actual value]

RMS position error is .0011 meters.

operation is obtained as shown in figure 5.10. In addition, the adaptive sliding controller is robust to significant parametric uncertainty with the ability to adapt on-line to changes in model parameters. This ability of the controller to adapt is best demonstrated by assuming the model parameters are initially unknown. Figures 5.11 and 5.12 demonstrate the convergence of model parameters when the parameters are initialized at zero. Parameter convergence was observed with a sinusoidal desired trajectory of frequency $0.3 \mathrm{~Hz}$ and amplitude of 0.2 meters. The parameters do not converge exactly to the actual values but are estimated only well enough to achieve a tracking error within the specified boundary layer. Adaptation ceases within the boundary layer, preventing long term drift.

The adaptive sliding controller is also robust to changes in model parameters as demonstrated by the tracking performance achieved when an obstruction is placed in the 
thruster duct outlet shown in figure 5.13. This is the same desired trajectory and obstruction used in the evaluation of the "pole" cancellation controller shown in figure 5.9 .

\subsection{Summary}

Any incorporation of thruster dynamics in the control of an underwater vehicle will improve the tracking performance significantly. The adaptive sliding controller demonstrates vehicle tracking performance superior to the other controllers evaluated.

Lead compensation is simplest to implement and could easily be incorporated in the analog vehicle control systems common on low-cost remotely operated vehicles. However, the lead compensation approach has performance limitations associated with thruster operation away from the lead network designed operating point.

The "pole" cancellation approach avoids the limitation associated with a single design operating point and performs well over the entire range of thruster operation. However, the "pole" cancellation approach lacks robust performance in the presence of significant model uncertainty. Both the lead compensation and "pole" cancellation controllers require knowledge of the precise dynamic characteristics of the individual thruster to be controlled.

With the addition of a propeller speed sensor to measure the thruster dynamic state, additional improvement in tracking performance can be obtained without a significant increase in computational requirements. The adaptive sliding controller suffers from none of the limitations observed in the lead compensation and "pole" cancellation controllers while offering a basis upon which even greater improvements in tracking performance might be obtained, for example, the incorporation of improved 
propeller speed sensing, improved adaptation schemes, and modified sliding mode control based on a time-varying boundary layer.

Through the on-line parameter estimation, the adaptive sliding controller implementation requires much less precise knowledge of individual thruster dynamic characteristics while being able to account for a variety of hydrodynamic effects encountered when the thruster is part of a real vehicle system. Though the hybrid simulation setup could not provide any verification, the adaptive sliding controller holds the promise of accounting for the observed thruster effects associated with cross-flow and mutual interference. 


\section{Chapter 6}

\section{EXPERIMENTAL SETUP}

This chapter consolidates the technical information on the test facility utilized in the model verification and controller evaluations.

\subsection{Thruster}

The typical thruster configuration consists of a propeller surrounded by a static shroud (or duct) coupled mechanically to an electric motor. The thruster used in the dynamic analysis presented in this thesis consists of a 10.2 inch diameter plastic propeller surrounded by a 5 inch long shroud coupled to a $1 / 3$ horsepower brushless DC motor.

The thruster motor is an 8-pole $3 \phi$ brushless DC motor with integral motor torque controller. For deep submergence applications, the motor is specially designed to be oil-filled to permit pressure compensation. A fixed DC voltage of 120 volts provides motor and logic power while a separate \pm 10 volt $\mathrm{DC}$ signal provides the torque command input. The analog torque command input voltage is generated by a 12 -bit D/A converter resident in the host computer/data logger. The input to the D/A converter was generated in software; off-line for the open-loop model verification and on-line as the output of the thruster controllers for their evaluation experiments. The motor has a rated input current of 3 amps at 120 volts DC though the attached propeller configuration can only load the 


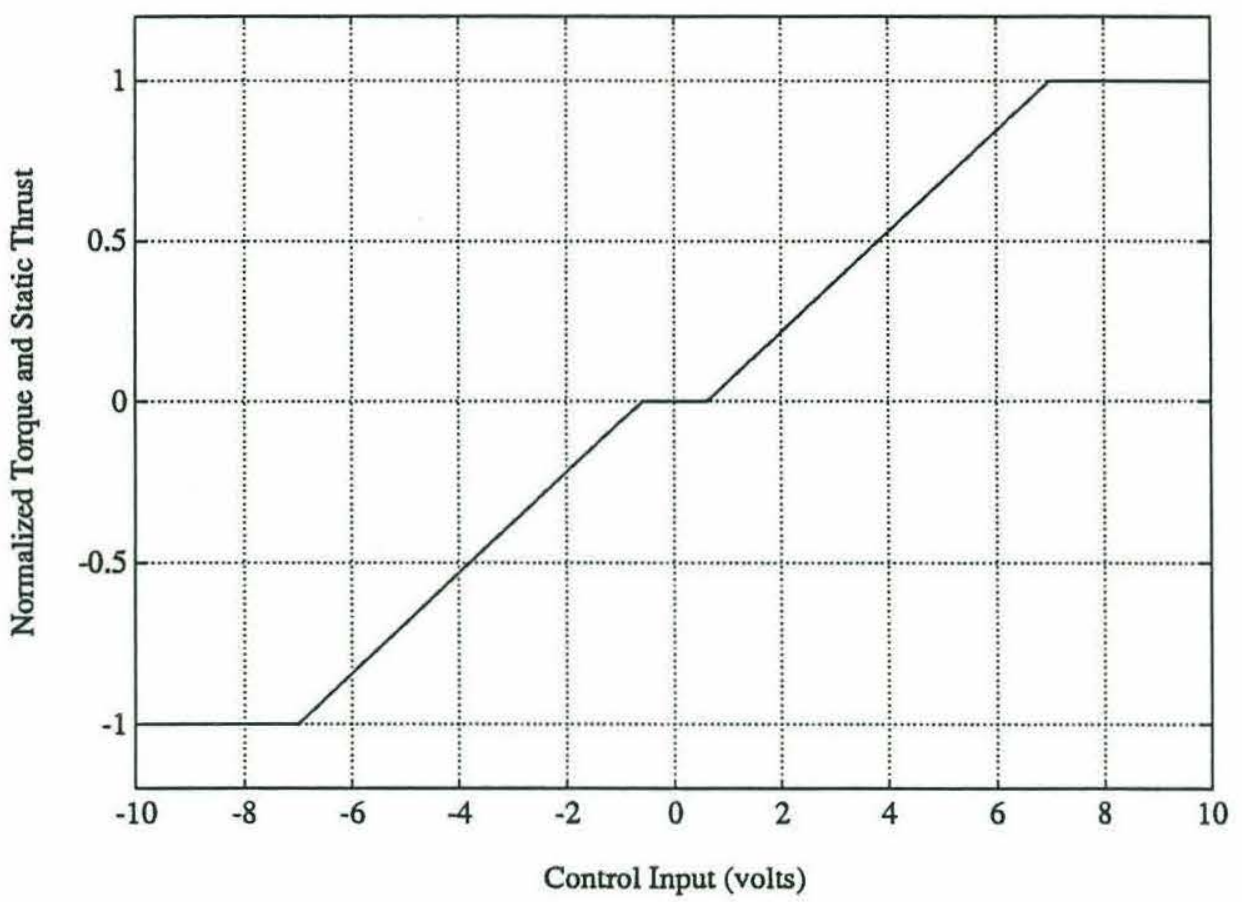

Figure 6.1: Relationship between torque, static thrust and input command. signal

motor to 2 amps at 120 volts DC with a propeller angular velocity of $775 \mathrm{rpm}$ and an output thrust of $28 \mathrm{lbf}$.

To avoid the effects of noise in the command input signal, a minimum signal level of \pm 0.6 volts is required to obtain a motor output. Motor torque and static thrust are linear with control voltage above the minimum. In the model and thruster relationships of previous chapters, the control voltage used is actually the voltage above the \pm 0.6 volts. As a result of the propeller loading and low voltage limits, the motor has an effective command input range of \pm 0.6 to \pm 7.2 volts. This relationship is presented graphically in figure 6.1. 


\subsection{Sensors}

\subsubsection{Propeller Speed Sensor}

The propeller angular velocity was selected as the primary measure of the thruster dynamic state, as it could be sensed most easily, and over a wide dynamic range. Previous efforts to instrument the thruster with hall effect sensors had been thwarted by interference from the motor magnetic field, therefore a new sensor was developed using electro-optic components. Electro-optic sensors have the advantage of wide dynamic range, high noise immunity and compact physical configuration but have the disadvantage of requiring encapsulation from submergence pressure.

The sensor was designed to be unobtrusive, requiring a minimum of modification to the existing hardware to install. The electro-optic microsensor consists of an infrared LED and a phototransistor. The phototransistor conducts when illuminated by the reflected LED output. The electro-optic microsensor is mounted in the thruster duct stator hub and sealed from submergence pressure behind a plexiglas cover. The reflective pattern is painted on a polished aluminum disk which is mounted on the propeller face.

The pattern consists of 64 alternating reflective and nonreflective segments. Figure 6.2 is a schematic representation of the propeller speed sensor configuration.

The output of the electro-optic microsensor is a series of pulses, each of which represents an arc length of propeller travel. This output signal is processed through a Schmidt-trigger to form a uniform square wave pulse train which is then input to a multifunction counter-timer which provides accumulated counts as well as a timebase to the host computer/data logger. The output of the speed sensor at this point is actually propeller "position". For the model verification this signal was recorded directly, differentiated and filtered off-line to generate the propeller angular velocity. The 


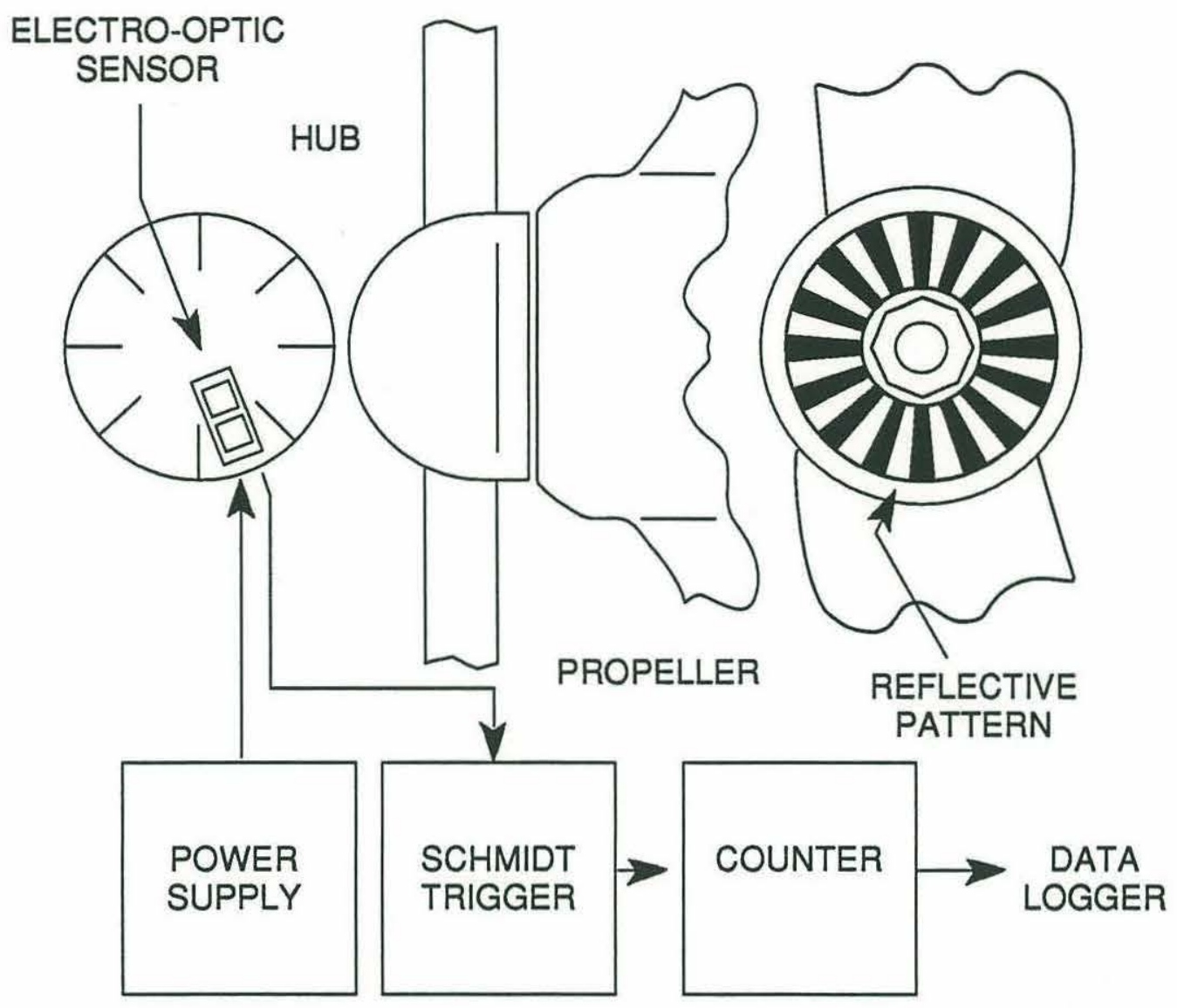

Figure 6.2: Schematic representation of the propeller speed sensor configuration.

controller applications required real-time propeller angular velocity information which was obtained using the measured counts per sample interval processed through a digital third-order elliptic filter to smooth the signal with minimum phase shift.

The speed sensor as designed and implemented cannot resolve the direction of propeller rotation. Direction of rotation for the real-time experiments was determined from the sign of the measured thrust. Of note, the phase lag between the propeller angular velocity and the input torque command precludes use of the sign of the input to determine the direction of rotation, demonstrating the prominence of thruster dynamics. In vehicle applications, the speed sensor configuration will require a second electro-optic 
microsensor and a modified reflective pattern to resolve the direction of propeller rotation.

\subsubsection{Thrust Sensor}

Thrust is sensed by a S-type load cell rated at $50 \mathrm{lbf}$ (compression or tension) full-scale, located between the thruster motor and the supporting test stand. Excitation, calibration and the output signal conditioning of the integral strain gage is provided by a sensor coprocessor resident in the host computer/data logger.

\subsubsection{Fluid Velocity Sensor}

The fluid velocity at the outlet of the thruster duct was measured by an analog electromagnetic (EM) current meter. The EM current meter has a low dynamic range and therefore was used to measure steady-state thruster performance only. The output meter deflection required manual recording. The EM current sensor was mounted at the thruster duct outlet. Varying the mounting configuration indicated the sensor was quite sensitive to orientation as well as distance from the outlet producing relatively low precision results.

\subsection{Test Stand}

The instrumented thruster was mounted to a steel frame test stand and placed in a 1000 gallon pool. The thruster weight was supported by a pinned rod, allowing only the generated thrust to contribute to the force applied to the load cell. This arrangement minimized the structures between the thruster, load cell, and rigid test stand to preclude 


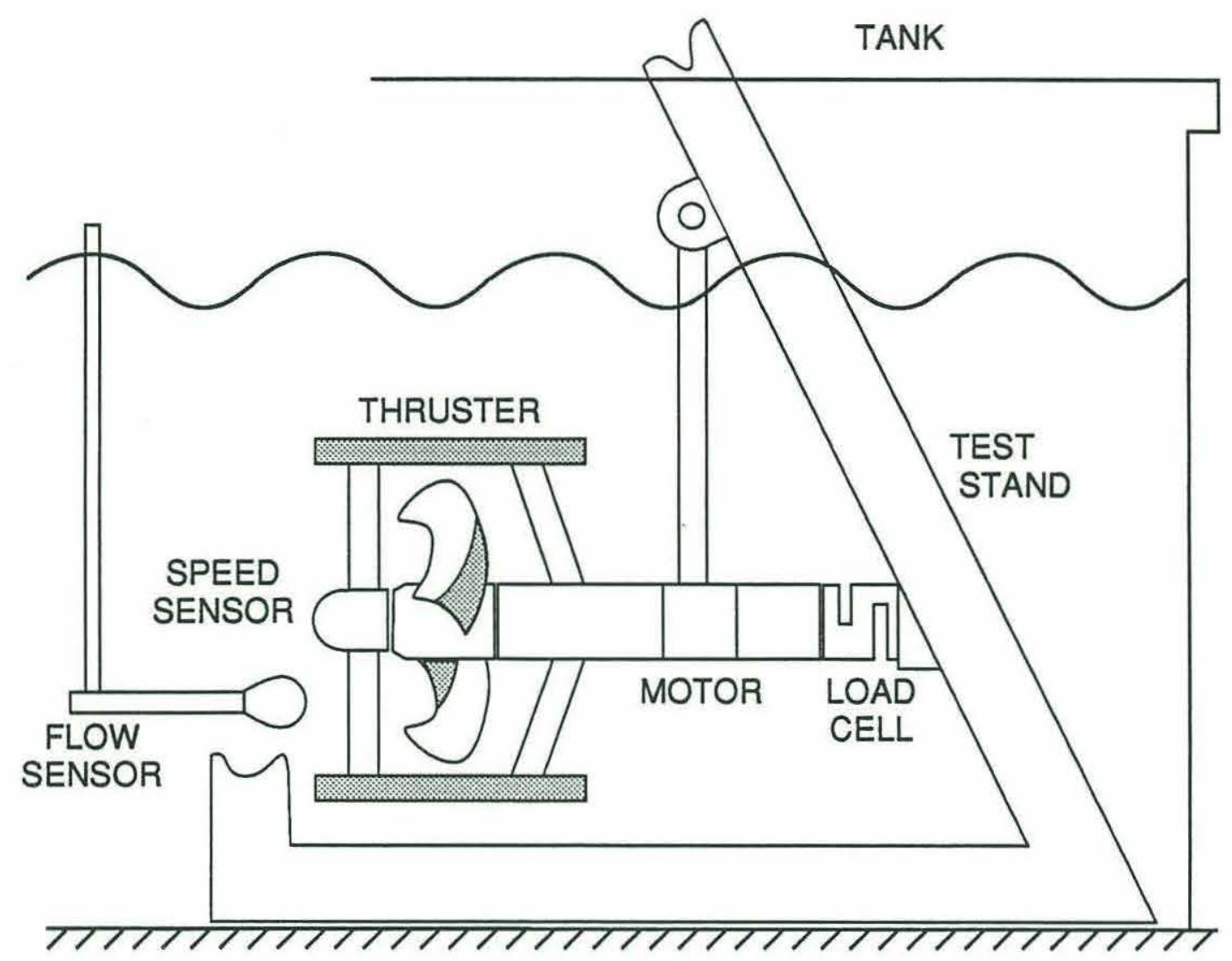

Figure 6.3: Schematic representation of the complete thruster test facility.

the introduction of structural resonances in the thrust measurement. The complete thruster test facility is presented schematically in figure 6.3.

\subsection{Summary}

The experimental setup satisfied all the design requirements and objectives, providing a flexible testbed for thruster dynamic response measurements as well as thruster controller evaluations. The development and construction of the test facility 
further addressed many of the implementation issues likely to be encountered in an actual vehicle application requiring thruster dynamic measurements. 


\section{Chapter 7}

\section{SUMMARY, CONCLUSIONS AND RECOMMENDATIONS}

Motivated by the precise vehicle position control required for repeatable survey and coordinated vehicle/manipulator operation, attention was focused on the dynamics associated with the actuator found on most underwater vehicles, the thruster. Using an energy-based, physical system approach, a dynamic model of the thruster was developed using the propeller angular velocity as the thruster state variable. A thruster test facility was constructed including an instrumented thruster, and the developed model was verified to satisfy the observable static and dynamic relations. Numerical values were obtained for the model parameters and compared against thruster geometric characteristics, confirming that in addition to the proper structure, the thruster dynamic model represents an accurate physical interpretation.

Controller design was approached from a practical standpoint, examining analog and simple digital techniques based on observations of the thruster step response as well as a complete model-based approach which incorporated the nonlinear thruster dynamics into a controller robust to significant model uncertainty. The controller designs were implemented and evaluated using a hybrid simulation technique utilizing an actual thruster fitted with appropriate sensors as the actuator for a simulated one degree-offreedom position-controlled vehicle. This hybrid simulation allowed controller 
evaluation and comparison to be based directly on the effects on vehicle tracking performance. The evaluation results demonstrated that the incorporation of thruster dynamics in the control of underwater vehicles will improve the tracking performance significantly. Additionally, an adaptive sliding controller representing the complete model-based approach, indicated additional gains in tracking performance could be achieved with proper feedback and control of the thruster dynamic state.

The established adaptive controller structure is based on the premise that there be no adaptation to that which can be modeled but adapt only to the thruster dynamic effects which cannot be simply modeled such as cross-flow and mutual interference.

For the controller evaluations conducted, an ideal vehicle model was utilized. A more complete evaluation should be conducted which includes the effects of common nonidealities in the vehicle and its position-controller such as pure time delays and measurement noise. In particular, the effects on the desired thrust rate signal should be evaluated, as this signal is important to the tracking performance of both the "pole" cancellation and adaptive sliding controllers.

The sliding controller design presented can be enhanced to maximize the available bandwidth of the thruster. By including the state dependence of the dynamic uncertainty, the boundary layer may be made time-varying, i.e., narrow when dynamic uncertainty is low and wide when dynamic uncertainty is high.

The adaptive sliding controller provides on-line adaptation to changing or uncertain model parameters within the model structure representation using the propeller angular velocity, $\omega$ as the dynamic state. Adaptation to changes in the relation, thrust = $\mathrm{C}_{\mathrm{t}} \omega|\omega|$ cannot be accomplished however at the thruster control level. Integration of the thruster controller within the vehicle position controller would support adaptation to the above relation as well as composite adaptation schemes which generate parameter estimates driven by tracking and prediction errors. 
The development of the thruster dynamic model and subsequent controller design and evaluation provided insight into thruster design specifications. The importance of measuring and controlling the thruster dynamic state is demonstrated. With this in mind, a speed-controlled motor may provide better dynamic control of a thruster, if the controller basic design as well as its implementation explicitly utilize the thruster dynamic structure. Thruster propeller and duct selection should proceed with an understanding of their effects on the thruster dynamic response. For instance, the parametric form for the apparent first-order thruster lag coefficient, derived in chapter 4 is,

$$
\lambda_{\mathrm{v}} \approx 2 \sqrt{\alpha \beta} \sqrt{\mathrm{V}_{\mathrm{C}}}
$$

which can be expanded in terms of thruster characteristics,

$$
\lambda_{\mathrm{v}} \approx 2\left\{\frac{\mathrm{A}}{2 \eta p \rho \mathrm{V}^{2}}\right\}^{1 / 2} \sqrt{\mathrm{V}_{\mathrm{C}}}
$$

where,

$\lambda_{\mathrm{v}}$ is the apparent thruster lag coefficient.

$\alpha$ and $\beta$ are thruster model parameters.

$\mathrm{V}_{\mathrm{C}}$ is the thruster command signal.

$\mathrm{A}$ is the thruster duct cross-sectional area.

$\eta$ is the propeller efficiency.

$\mathrm{p}$ is the propeller pitch.

$\rho$ is the fluid density.

$\mathrm{V}$ is the thruster involved fluid volume.

With these relations in mind, the thruster dynamics will be less prominent if $\lambda_{v}$ is maximized. This indicates that, for a constant involved volume, $\mathrm{V}$ the area of the duct and propeller should be maximized while minimizing the propeller pitch and efficiency. As a general rule, decreased pitch and efficiency are indicative of higher speed propellers. 
Though additional research and experimentation is required to confirm these results, the solutions presented are sufficiently developed to permit direct implementation on many ROV's, including JASON. The importance of incorporating thruster dynamics in the control of an underwater vehicle is clear. 


\section{References}

1. Yoerger, D. R. and J. B. Newman "Control of Remotely Operated Vehicles for Precise Survey." Proceedings ROV 1989, May 1989.

2. Beaman, J. J. and P. C. Breedveld "Physical Modeling With Eulerian Frames and Bond Graphs." ASME Journal of Dynamic Systems, Measurement and Control, Vol 110 (6), 1988.

3. Karnopp, D. C. and R. C. Rosenberg System Dynamics: A Unified Approach. New York: John Wiley and Sons, 1975.

4. Crandall, S. H., D. C. Karnopp, E. F. Kurtz and D. C. Pridmore-Brown Dynamics of Mechanical and Electromechanical Systems. New York: McGraw-Hill, 1968.

5. Ogata, K. Modern Control Engineering. Englewood Cliffs, NJ: Prentice Hall, 1970.

6. Slotine, J-J. E. and J. A. Coetsee "Adaptive Sliding Controller Synthesis for Nonlinear Systems." International Journal of Control, Vol 42 (6), 1986.

7. Asada, H. and J-J. E. Slotine. Robot Analysis and Control. New York: John Wiley and Sons, 1986.

8. Utkin, V.I. Sliding Modes and their Application in Variable Structure Systems. Moscow: MIR, 1974. 
9. Delonga, D. M. "A Control System Design Technique for Nonlinear Discrete Time Systems." Ph.D. Thesis MIT/WHOI, 1988. 